\title{
Direct observations of asteroid interior and regolith structure: Science measurement requirements
}

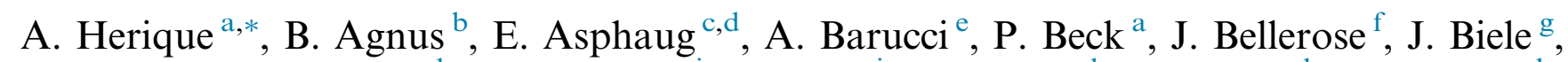
L. Bonal ${ }^{\mathrm{a}}$, P. Bousquet ${ }^{\text {h}}$, L. Bruzzone ${ }^{\mathrm{i}}$, C. Buck ${ }^{\mathrm{j}}$, I. Carnelli ${ }^{\mathrm{k}}$, A. Cheng ${ }^{1}$, V. Ciarletti ${ }^{\mathrm{b}}$,

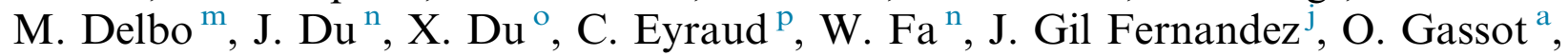
R. Granados-Alfaro ${ }^{\text {a }}$, S.F. Green ${ }^{\mathrm{q}}$, B. Grieger ${ }^{\mathrm{r}}$, J.T. Grundmann ${ }^{\mathrm{s}}$, J. Grygorczuk ${ }^{\mathrm{t}}$, R. Hahnel ${ }^{\circ}$, E. Heggy ${ }^{\text {u,f }}$, T-M. Ho ${ }^{\text {s }}$, O. Karatekin ${ }^{\text {v }}$, Y. Kasaba ${ }^{\text {w }}$, T. Kobayashi ${ }^{\mathrm{x}}$, W. Kofman ${ }^{\mathrm{a}, \mathrm{y}}$, C. Krause ${ }^{\mathrm{g}}$, A. Kumamoto ${ }^{\mathrm{w}}$, M. Küppers ${ }^{\mathrm{r}}$, M. Laabs ${ }^{\mathrm{o}}$, C. Lange ${ }^{\mathrm{s}}$, J. Lasue ${ }^{\text {zaa }}$, A.C. Levasseur-Regourd ${ }^{\text {ab }}$, A. Mallet ${ }^{\mathrm{h}}$, P. Michel ${ }^{\mathrm{m}}$, S. Mottola ${ }^{\mathrm{ac}}$, N. Murdoch ${ }^{\text {ad }}$, M. Mütze ${ }^{\circ}$, J. Oberst $^{\text {ac }}$, R. Orosei ${ }^{\text {ae }}$, D. Plettemeier ${ }^{\circ}$, S. Rochat ${ }^{a}$, R. RodriguezSuquet ${ }^{\text {h}}$, Y. Rogez ${ }^{\text {a }}$, P. Schaffer ${ }^{\circ}$, C. Snodgrass ${ }^{\text {q }}$, J-C. Souyris ${ }^{\text {h }}$, M. Tokarz ${ }^{\mathrm{t}}$, S. Ulamec ${ }^{\mathrm{g}}$, J-E. Wahlund ${ }^{\text {af }}$, S. Zine ${ }^{\mathrm{a}}$

${ }^{a}$ Univ. Grenoble Alpes, CNRS, IPAG, F-38000 Grenoble, France

${ }^{\mathrm{b}}$ LATMOS/IPSL, UVSQ Université Paris-Saclay, UPMC Univ. Paris 06, CNRS, F-78280 Guyancourt, France

${ }^{\mathrm{c}}$ Lunar and Planetary Laboratory, University of Arizona., Tucson, AZ 85721, USA

${ }^{\mathrm{d}}$ School of Earth and Space Exploration, Arizona State University, Tempe, AZ 85287, USA

${ }^{\mathrm{e}}$ LESIA, F-92195 Paris, France

${ }^{\mathrm{f}} J P L / C a l t e c h$, Pasadena, CA 91109, USA

${ }^{\mathrm{g}}$ German Aerospace Center (DLR), 51147 Cologne, Germany

${ }^{\mathrm{h}}$ CNES, F-3100 Toulouse, France

${ }^{\mathrm{i}}$ University of Trento, I-38123 Trento, Italy

${ }^{\mathrm{j}}$ ESA, ESTEC, 2200 AG Noordwijk, The Netherlands

${ }^{\mathrm{k}}$ ESA HQ, F-75015 Paris, France

${ }^{1}$ JHUIAPL, Laurel, MD 20723, USA

${ }^{\mathrm{m}}$ Université Côte d'Azur, Observatoire de la Côte d'Azur, CNRS, Laboratoire Lagrange, F-06000 Nice, France

${ }^{\mathrm{n}}$ IRSGIS, Peking University, Beijing 100871, China

${ }^{\circ}$ Technische Universität Dresden, 01069 Dresden, Germany

${ }^{\mathrm{p}}$ Aix Marseille Univ, CNRS, Centrale Marseille, Institut Fresnel, F-13013 Marseille, France

${ }^{\mathrm{q}}$ Open University, Milton Keynes MK7 6AA, UK

${ }^{\mathrm{r}}$ ESA, ESAC, 28692 Villanueva de la Cañada, Madrid, Spain

${ }^{\mathrm{s}}$ DLR German Aerospace Center, Institute of Space Systems, 28359 Bremen, Germany

${ }^{\mathrm{t}}$ ASTRONIKA Sp. Z o.o., 00-716 Warszawa, Poland

${ }^{\mathrm{u}}$ University of Southern California, Viterbi School of Engineering, Los Angeles, CA 90089-0241, USA

${ }^{\vee}$ Observatoire Royal de Belgique, 1180 Bruxelles, Belgium

${ }^{\mathrm{w}}$ Dep. Geophysics, Tohoku University, Sendai, Miyagi 980-8578, Japan

${ }^{\mathrm{x}}$ Geological Research Center, Korea Institute of Geoscience and Mineral Resources, Daejeon 34132, Republic of Korea

${ }^{\mathrm{y}}$ Space Research Centre, PAS, 00-716 Warsaw, Poland

${ }^{\mathrm{z}}$ Université de Toulouse, UPS-OMP, IRAP, Toulouse, France

${ }^{\text {aa }}$ CNRS, IRAP, 9 Av. colonel Roche, BP 44346, F-31028 Toulouse cedex 4, France

${ }^{\mathrm{ab}}$ UPMC (Sorbonne Univ.), UVSQ (UPSay), CNRSIINSU, LATMOS-IPSL, F-75252 Paris, France

${ }^{\mathrm{ac}}$ German Aerospace Center (DLR), Institute of Planetary Research, 12489 Berlin, Germany

* Corresponding author.

E-mail address: Alain.herique@univ-grenoble-alpes.fr (A. Herique), . 


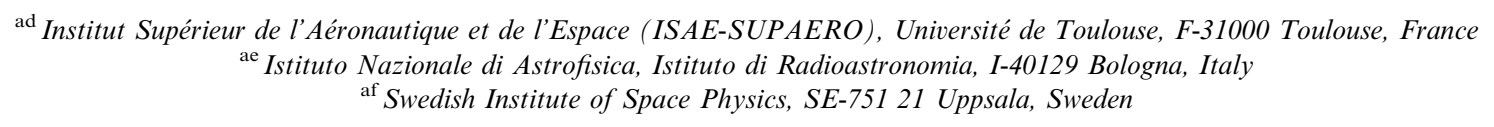

Received 28 April 2017; received in revised form 13 October 2017; accepted 16 October 2017

Available online 26 October 2017

\begin{abstract}
Our knowledge of the internal structure of asteroids is, so far, indirect - relying entirely on inferences from remote sensing observations of the surface, and theoretical modeling of formation and evolution. What are the bulk properties of the regolith and deep interior? And what are the physical processes that shape asteroid internal structures? Is the composition and size distribution observed on the surface representative of the bulk? These questions are crucial to understand small bodies' history from accretion in the early Solar System to the present, and direct measurements are needed to answer these questions for the benefit of science as well as for planetary defense or exploration.

Radar is one of the main instruments capable of sounding asteroids to characterize internal structure from sub-meter to global scale. In this paper, we review the science case for direct observation of the deep internal structure and regolith of a rocky asteroid of kilometer size or smaller. We establish the requirements and model dielectric properties of asteroids to outline a possible instrument suite, and highlight the capabilities of radar instrumentation to achieve these observations. We then review the expected science return including secondary objectives contributing to the determination of the gravitational field, the shape model, and the dynamical state. This work is largely inherited from MarcoPolo-R and AIDA/AIM studies.
\end{abstract}

(C) 2017 COSPAR. Published by Elsevier Ltd. All rights reserved.

Keywords: Near-earth asteroid; Radar tomography; Dielectric properties; Asteroid deep internal structure; Asteroid regolith

\section{Introduction}

Asteroids are fundamental to our understanding of the formation and history of our Solar System. Their diverse population ranges from primitive asteroids, whose materials have experienced the least processing since their condensation from the early solar nebula, to metallic asteroids widely interpreted as fragments of collisionally disrupted differentiated planetesimals. The asteroid population tells us the story of planetary accretion in the early Solar System and its evolution (Chambers and Wetherill, 1998; Morbidelli et al., 2000; Blum and Wurm, 2008). Consequently, a fleet of spacecraft is in operation, in cruise or under development, to observe asteroids: Dawn (NASA, launched 2007) rendezvous mission to 4 Vesta in 20112012 and 1 Ceres in 2015-2017 (Russell et al., 2015), Hayabusa2 (JAXA, 2014) sample-return mission to the C-type asteroid 162173 Ryugu in 2018-2019 (Tsuda et al., 2016), OSIRIS-REx (NASA, 2016) sample-return mission to the B-type asteroid 101955 Bennu in 2018-2021 (Lauretta, 2015) and the recently selected NASA/Discovery missions, Lucy, expected to be launched in 2021 to visit Jupiter's Trojan asteroids, and Psyche to the metallic asteroid 16 Psyche, expected to be launched in 2022.

In the past (see, e.g., Barucci et al., 2011), a few missions were dedicated to asteroids as a prime objective, e.g., NEAR-Shoemaker (NASA, 1996) to 433 Eros in 20002001, Deep Space 1 (NASA, 1998) to 9969 Braille in 1999 and Hayabusa (JAXA, 2003) to 25143 Itokawa in 2005, or as secondary objectives like Galileo (NASA,
1989) to fly-by 951 Gaspra in 1991 and 243 Ida in 1993, NEAR-Shoemaker (NASA, 1996) to (243) Mathilde in 1997, Stardust (NASA, 1999) to 5535 Annefranck in 2002, Rosetta (ESA, 2004) to 2867 Steins in 2008 and 21 Lutetia in 2010, and finally Chang'e 2 (CNSA, 2010) to 4179 Toutatis in 2012 (Zhu et al., 2014).

It is remarkable to note that, despite the fairly large number of missions to asteroids, their internal structure is still poorly known and has never been measured directly for any asteroid. Therefore, important questions do not have a definite answer, even for asteroids whose bulk density has been estimated. For instance, are most asteroids monolithic pieces of rock, or are they rubble piles, i.e. aggregates of boulders held together by gravity? And is there a size threshold between these two structures? What is the porosity of a given asteroid, and is it homogeneous or multi-scale? What is the typical size of its constituent blocks? Are these blocks homogeneous or heterogeneous? What is the average thickness of the regolith covering the surface and how is it distributed? These are key to understanding the evolution of asteroids from their formation in the primitive solar nebula. However, our knowledge is only based on mass deduced from spacecraft fly-by trajectories (radioscience) and on inferences from remote sensing observations of the surface combined with theoretical modeling.

Because these questions are so crucial to understand and model asteroid accretion and evolution history, several missions have recently been proposed or are currently under selection to embed instrument suites dedicated to sub-surface sounding of asteroids. These instruments are 
based on radar or seismology techniques, that is, analysis of the controlled propagation of electromagnetic or acoustic waves throughout the volume. In the context of the 5th Medium Class (M5) ESA Cosmic Vision call, the Castalia mission to a Main-belt Comet (Snodgrass et al., 2018), the Heavy Metal mission to Psyche (Wahlund et al., 2016) and the MarcoPolo-M5 sample return mission to a D-type asteroid (Franchi et al., 2017), each include an instrument aimed at internal structure measurements. Such an instrument was also considered for the AIDA technology demonstration mission, composed of a kinetic impactor DART (NASA, Cheng et al., 2016) and of an observing spacecraft AIM (ESA, Michel et al., 2016) to test a deflection scenario and to study the binary near-Earth asteroid system, 65803 Didymos. Unfortunately, the European AIM component was not funded by ESA Member States during the Ministerial council in 2016. A reduced version of AIM, currently under study, does not include internal structure direct measurements (Michel et al., 2018) but the concept is still valid for possible further options to achieve the original objectives.

In this paper, we present the scientific issues for direct observation, using proven radar techniques, of the internal structure of a rocky asteroid of kilometer size or smaller. We establish the requirements for a future instrument suite, expose the capabilities of radar instrumentation to achieve these observations, and outline a possible instrument suite. We then review the expected science return including secondary objectives. This work is largely inherited from the Fantina instrument suite (Herique et al., 2012) proposed for the MarcoPolo-R mission, which reached the final selection for the ESA M3-Class ESA Cosmic Vision program opportunity (Barucci et al., 2012), as well as for the AIDA/AIM phase A/B1 study at ESA.

\section{Why are direct observations of asteroid interiors crucial?}

\subsection{Deep interior and collisional history}

So far, the interior structure of an asteroid has never been directly measured. There is no way, from classical optical remote sensing observations, to determine whether a body is monolithic (i.e., like a single piece of rock) or whether it is a gravitational aggregate (also called a "rubble pile"). Similarly, there is no optical technique to estimate a small body's porosity, nor the porosity distribution in the form of small (micro-) and large (macro-) porosity or voids. Intuition and inference are applied, such as those based on spin rates and shapes, along with elaborate simulations and modeling, leading to a situation in which a lot of science is built upon hypotheses. In many cases even our intuition is insufficient to come up with plausible explanations. For instance, the bulk density of less than $1.5 \mathrm{~g} \mathrm{~cm}^{-3}$ of asteroid 101955 Bennu, the target of the OSIRIS-REx mission (Chesley et al., 2012, 2014), is still hard to interpret. What could possibly be the implication of such a low density for the internal structure of the asteroid, when it is presumably a primitive chondrite with material density greater than $3 \mathrm{~g} \mathrm{~cm}^{-3}$ ?

Collisional evolution models and measured bulk densities of some asteroids suggest that a significant number of small asteroids (typically smaller than $50 \mathrm{~km}$ and larger than a few hundred meters in diameter) have a rubble pile structure:

For example, Itokawa, the near-Earth stony asteroid (Stype) visited by the JAXA Hayabusa spacecraft in 2005, has a size of about $320 \mathrm{~m}$ and a relatively low bulk density $\left(1.9 \pm 0.13 \mathrm{~g} \mathrm{~cm}^{-3}\right.$, Fujiwara et al., $2006 ; 1.95 \pm 0.14 \mathrm{~g}$ $\mathrm{cm}^{-3}$ Abe et al., 2006) as compared to its meteorite analog (between 3 and $4 \mathrm{~g} \mathrm{~cm}^{-3}$ for ordinary chondrites; Britt and Consolmagno, 2003). The analogy with ordinary chondrites was confirmed by the returned sample (the first ever returned samples from an asteroid), which have a similar composition to LL chondrites (Nakamura et al., 2011). This latter result, together with images showing that its granular surface is covered by large pieces of debris, as well as simulations of catastrophic disruption and reaccumulation (Michel and Richardson, 2013), suggest that Itokawa is indeed a rubble pile (Fujiwara et al., 2006). From surface images, it seems to be composed of 100 $200 \mathrm{~m}$ blocks and to be covered by a thin regolith mainly composed of gravels and pebbles (Barnouin-Jha et al., 2008 and Fig. 1). However, each of these larger blocks can be a monolith or an aggregate of smaller blocks, pebbles or gravels, and other internal structures can be invoked to explain the same observable features. For example, following the suggestion (Scheeres and Gaskell, 2008) that the non-detection of the YORP effect on Itokawa could be the result of a non-uniform mass distribution, Lowry et al. (2014) inferred mean densities of $1.75 \pm$ $0.11 \mathrm{~g} \mathrm{~cm}^{-3}$ and $2.85 \pm 0.50 \mathrm{~g} \mathrm{~cm}^{-3}$ for two components of the asteroid to reconcile their YORP measurement. This would imply one rubble pile lobe, attached to a monolithic lobe, something that is not at all easily explained by modeling. However, there are possible alternative explanations for the discrepant data, which demonstrates the need for direct measurements.

In the same way, Mathilde, the main belt primitive asteroid (C-type) observed by the NASA NEARShoemaker spacecraft during a fly-by in 1997, has a size of about $50 \mathrm{~km}$ and a low bulk density $(1.3 \pm 0.2 \mathrm{~g}$ $\mathrm{cm}^{-3}$ ). Its porosity is larger than $50 \%$, as derived from a comparison between its density and that of meteorite analogues (Yeomans et al., 1997). A rubble pile structure with voids between blocks (e.g., Asphaug et al., 2002) and possibly micro-porosity within the individual blocks can explain this difference (e.g., Housen et al., 1999).

And finally. Eros, which was visited by the NASA NEAR-Shoemaker spacecraft in 2000-2001, can either be interpreted as a shattered monolithic block (Cheng, 2009) or as a sand pile (Asphaug, 2009) based on surface observations.

Only a geophysical sounding investigation will allow us to discriminate between two possible structures currently 

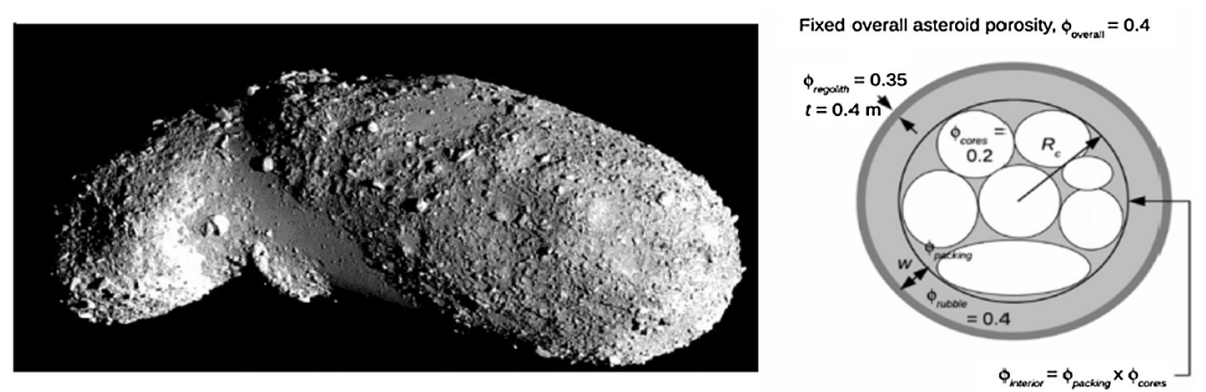

Fig. 1. Itokawa (left from JAXA Fujiwara et al., 2006); is a candidate example of the aggregate or rubble-pile structural model (right from Barnouin-Jha et al., 2008).

based solely on surface observations or global measurements and meteorite analogues. As we have no a priori robust idea of the detailed internal structure of any asteroid, the results of interior sounding may require a revision of our current ideas on the origin of such small bodies and on how to relate surface properties to internal ones. Such information is crucial for our understanding and modeling of the evolution of an asteroid, beginning with its formation in the primitive solar nebula. For example, the internal structure of an asteroid determines its response to impacts by other small bodies and, consequently, its collisional lifetime. As models of the collisional evolution of small body populations over the history of the Solar System are dependent on the assumptions made on their internal structure, uncertainties in these properties can result in significant differences in our understanding of planetary accretion and collisional history.

Numerical models of the collisional evolution of asteroids indicate that the vast majority of small asteroids are of secondary origin, i.e., they are fragments of larger parent bodies disrupted by collisions with smaller objects (Asphaug, 2009; Cheng, 2004). Numerical simulations indicate that fragments larger than a few hundreds of meters are produced by gravitational re-accumulation of smaller fragments from the original body (Benz and Asphaug, 1999). This modeling work suggests that most asteroids smaller than $\sim 50 \mathrm{~km}$ in size (that is the vast majority of asteroids) should be rubble piles or heavily shattered bodies. Moreover, there should be a link between the size distribution of the blocks building up the asteroid and the past and future fragmentation of the object. Consequently, direct information on the size of the building blocks would provide crucial information to understand the collisional history of the asteroid and possibly the linked history of any retrieved sample. Note that in the range of impact energies involved in the events generating those bodies, except for extreme (rare) cases, heat effects are expected to be negligible and to affect at most a small region close to the impact point (Jutzi et al., 2015). Thus, the composition of the material constituting those bodies should not be strongly altered by their collisional history.

Rubble pile structures are also invoked to explain the large fraction of binary systems $(15 \%)$ observed in near-
Earth asteroid populations (Margot et al., 2002). Arguably, the most realistic formation scenario for small binary systems in the near-Earth asteroid population and in the inner main belt is the spin-up of a rubble pile due to the thermal YORP effect (thermal torques generated by the heating of the object through the absorption of sunlight, and the reradiation of this energy as heat, Rubincam, 2000) beyond the spin frequency at which centrifugal force causes a loss of particles that can then re-accrete to form a small satellite (Walsh et al., 2008). This scenario also allows reproducing the observed oblate spheroidal shape (also called top shape) of the primary of small binary systems, caused by the reshaping of the primary rubble pile asteroid as a result of spin-up and consequent mass loss. In this model, the satellite is slowly built in orbit by repeating massshedding events. Alternatively, the YORP effect may cause also a fission of the primary body, eventually also leading to a satellite (Jacobson and Scheeres, 2011). In this case, the mass loss is a more singular catastrophic event (a fission) and later processing of this lost mass is invoked for the formation of the equatorial ridge of the primary giving it its top shape. Both scenarios require an aggregate to start with, but the detailed structure of the aggregate and that of the resulting two components are quite different in the two scenarios. In order to better constrain the mechanism that forms binaries and discriminate between the two formation models for binaries, we need to obtain direct information on the internal structure of an actual asteroid. Another scenario, which best explains binary systems consisting of a very large primary, not necessarily with a top shape, and a very small satellite is the formation of a satellite during a catastrophic disruption when the ejected debris reaccumulates and some of them become two gravitationally bound bodies (Durda et al., 2004; Michel et al., 2001).

\subsection{Regolith formation and dynamics}

So far, only the surfaces of asteroids have been observed by spacecraft. It was found that these airless bodies are covered by a blanket of soil, broken rocks, dust, and other fine granular materials that are collectively called regolith (see, e.g., Murdoch et al., 2015). The fact that regolith is ubiquitous on asteroid surfaces is supported by thermal infrared 
observations (Delbo et al., 2007, 2015) which indicate that asteroid thermal inertia has values generally well below those expected from bare rocks. Regolith properties can differ dramatically from one small body to another, even when they have similar composition. For instance, the regolith on the $23 \mathrm{~km}$-size asteroid Eros observed by NEARShoemaker consists of fine grained material. In the same spectral class, the regolith on the $320 \mathrm{~m}$-size Itokawa, as observed by Hayabusa, mostly consists of widely distributed gravels and pebbles with spatial segregation depending on the size-distribution of the constitutive grain. Landslides features have been observed on Lutetia by Rosetta and on Vesta by Dawn. However, in all of these cases, the depth and structure of the regolith lack direct measurements and are only inferred from surface observations.

Direct measurements of the regolith depth and structure, and of its lateral variations, would give better constraints on the process of regolith formation and evolution on asteroid surfaces, and help understand how such small bodies can retain loose material while their effective surface gravities are really low in magnitude. The formation and dynamics of granular materials in low-gravity environments has become an important area of research, given its importance to the interpretation of spacecraft mission data and preparation for spacecraft missions aimed at interacting with asteroid surfaces.

Having direct information would help improve our understanding and refine our ability to model asteroid surfaces. Regolith properties depend on the regolith formation process. At least two main processes have been invoked to generate regolith, which are not necessarily exclusive: retention of ejecta resulting from impact cratering (see, e.g., Richardson, 2011) and thermal fragmentation (see, e.g., Delbo et al., 2014). Having direct measurements of the regolith properties (e.g. size distribution) and abundance (depth and stratigraphy) will allow us to better constrain its formation and evolution, including transport in a low-gravity environment. There is no way we can answer such questions about small bodies' surface material without having direct access to detailed characteristics, such as regolith thickness, size distribution and possibly variation as a function of surface location. These measurements will also allow as us to infer other regolith properties, such as frictional and cohesive properties, that are important for modeling and interpretation of surface features.

Asteroid surface processes are largely dependent on the material properties at depth. Mass migration, size segregation or landslides in low gravity are highly dependent on the material properties of the subsurface. By combining observations of the surface and interior with numerical modeling, we can constrain those properties, to more accurately predict the effects of surface processes on small bodies and the interaction of future spacecraft with their surfaces.

Because of its potentially high porosity, the regolith is also a thermally insulating layer (Schorghofer, 2008). The knowledge of the regolith thickness and its lateral variation is the only way to better model the thermal state of the asteroid surface and therefore the magnitude of the Yarkovsky thermal effect and the YORP effect which are key for the long term orbit prediction, especially applied to risk mitigation of potentially hazardous objects.

In the case of space missions aimed at deflecting an asteroid, the regolith structure and depth can have a big influence on the efficiency of the various deflection techniques. All techniques relying on anchoring or similar surface interaction need some knowledge of the regolith properties. In the case of a kinetic impactor, depending on the depth and structure of the regolith layer, the shock wave may be transmitted more or less efficiently, which in turn will influence the momentum transfer efficiency.

\subsection{The constitutive material}

Probing of the interior is key to understanding structures and dynamical processes. It is also crucial for determining material composition and mineralogy. Space weathering (the cumulative effects of solar radiation, energetic particles and micrometeoroid collisions) alters the uppermost few microns of asteroid surface materials while thermal cycling affects greater depths. Therefore, surface properties may not be representative of the interior, in terms of mineralogy and chemical composition.

Assessing the homogeneity or heterogeneity within the first meter below the surface is essential since the surface has experienced thermal fracturing and radiative heating by the Sun. Note that a number of asteroids do contain water-ice (Campins et al., 2010), that is likely present at the surface in permanently shadowed regions, and in the close subsurface (see, e.g. Prettyman et al., 2017 on Ceres). As a response to solar heating a number of compounds can be degraded or lost to space, including some of the organic materials found in chondritic meteorites and interplanetary dust particles. In the case of objects with a very low perihelion distance $(<0.1 \mathrm{AU})$ the surface temperature can become high enough to degrade some of the mineral compounds, which can decrepitate. This includes iron oxides and some of the phyllosilicates found in primitive meteorites (Garenne et al., 2014). Assessing the vertical degree of homogeneity and whether a degradation gradient is present in the first meter is crucial for these compounds, particularly in the framework of a sample return mission.

The surfaces of grains exposed to solar radiation during millions of years are altered by space weathering. A wellknown and major consequence is the flattening of their optical and near-infrared spectral signature, resulting in difficulties with comparison with meteorite spectra using remote-sensing observations. For bodies covered with regolith, this effect can be limited by resurfacing processes induced by grain mobility and YORP acceleration. On the other hand, the surface non-representability could also come from grain mobility mechanisms and from the regolith origin by itself (at least for the scenario based on gravitational accretion): these processes can induce grain 
segregation in size and in composition and a fraction of exogenous materials could also be present. Optical remote sensing observations of the surface only could introduce substantial bias and limit significantly the scope of the results.

Similar bias is expected in meteorite analyses. Except for Itokawa's samples, ground-based analyses in laboratories of asteroid materials are so far limited to the randomly selected meteorites that have survived the voyage through space, the violent passage through Earth's atmosphere, and residence upon the surface. How representative meteorites are of pristine asteroid materials is still a matter of debate. A basic knowledge of surface composition of asteroids may nevertheless be derived from albedo and spectral measurements, although with large remaining uncertainties. An extrapolation to the interior is then only feasible as long as one assumes that the effects of impact processing and solar wind irradiation have not significantly modified the surface albedo and spectral properties.

The limits of such an approach, regarding the possible presence of heterogeneities in a single body, are illustrated by the fall of the meter-sized asteroid 2008 TC3. It was the first time a small body fragment was spectrally observed prior to its atmospheric break-up into smaller pieces that eventually reached Earth's surface. It was originally identified as an F-type object (relatively similar to C-type); however, surprisingly, the recovered meteorites were found to be friable breccia, each with its own lithology, ranging from ureilite to enstatite, ordinary and carbonaceous chondrite clasts (Horstmann and Bischoff, 2010; Jenniskens et al., 2009). Such a variety of meteorite types originating from a single body is unprecedented.

To bypass such potential biases we need a physical characterization of the subsurface material with the context from surface measurements and supporting laboratory analyses. Sample return missions, such as OSIRIS-REx, and Hayabusa and Hayabusa2, offer a unique opportunity to obtain ground truth for the mineralogy, the chemistry and the physical structure of the surface material of a primitive asteroid. Indeed, such missions offer a unique way to connect ground truth (the analysis of retrieved samples) with asteroid and meteorite observations. The laboratory characterization of returned samples is crucial to determining how representative they are of the parent object (as observed from the spacecraft and from the Earth). A previous sample return mission, Stardust, was a tremendous success in retrieving mineral grains from the dust particles within the coma of a comet, 81P/Wild 2, for analysis on Earth (Brownlee et al., 2006; Zolensky et al., 2006). However, firm conclusions about the bulk composition of the comet, based on the analysis of these samples, is not possible due to questions of the representativeness of the samples, possible segregation processes in the nucleus, and possible sampling bias during collection at $6.1 \mathrm{~km} \mathrm{~s}^{-1}$. Consequently, we need a geologic map in 3D to support sample return missions not only to understand the context but also for sampling site selection.

\section{How do we characterize deep interior and near surface regolith?}

Which instruments on an asteroid mission should aim at understanding the physical properties of the target asteroid by determining the characteristic sizes of its constituents, ranging from the sub-meter size to that of the whole body? Radar is the most mature technique capable of achieving these science objectives. Which radar concepts and requirements should achieve these science objectives? A monostatic radar configuration and a bistatic one (Fig. 3) should be considered in the instrument trade-off to choose the frequency and the bandwidth.

The internal structure of asteroids, and even the nearsubsurface structure, is almost unknown. This structure could drastically differ from one mission target to another and its direct observation is our major goal. Two endmember models can be imagined. At one extreme, is a monolithic body, which is well-characterized by its average composition and micro-porosity, as well as by an internal heterogeneity (stratigraphy, accretion or collisional metamorphism, hydration, fracturing) that will be revealed in radar images of the internal structure. At the other extreme is a body composed of an aggregate of a large number of fragments and other debris that is best characterized by the size distribution and composition of the constituent blocks, and their spatial heterogeneity (caused by segregation from impacts and re-accretion, as well as mass redistribution by YORP effect). Radar tomography can provide this characterization.

Fig. 2 summarizes the possible size range of asteroidal constituents. Asteroids are likely to be composed of a variety of different-sized constituents, given the results from previous spacecraft observations of asteroids. From sur-

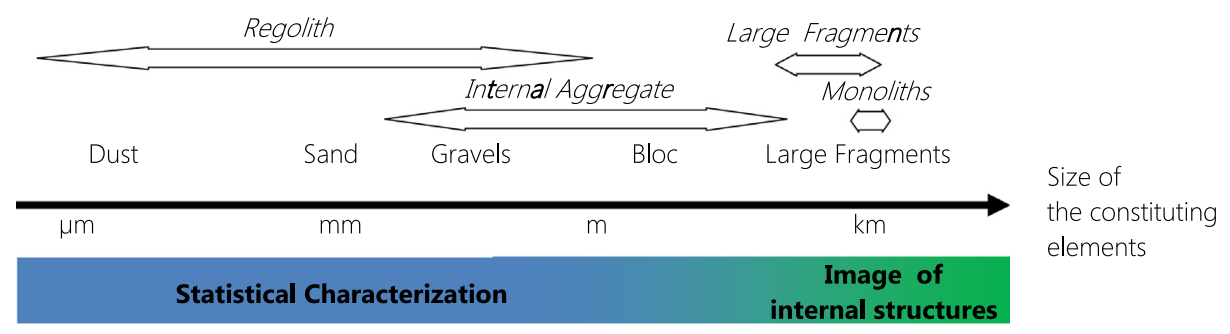

Fig. 2. Asteroid structures, size distribution and tomography approach. 

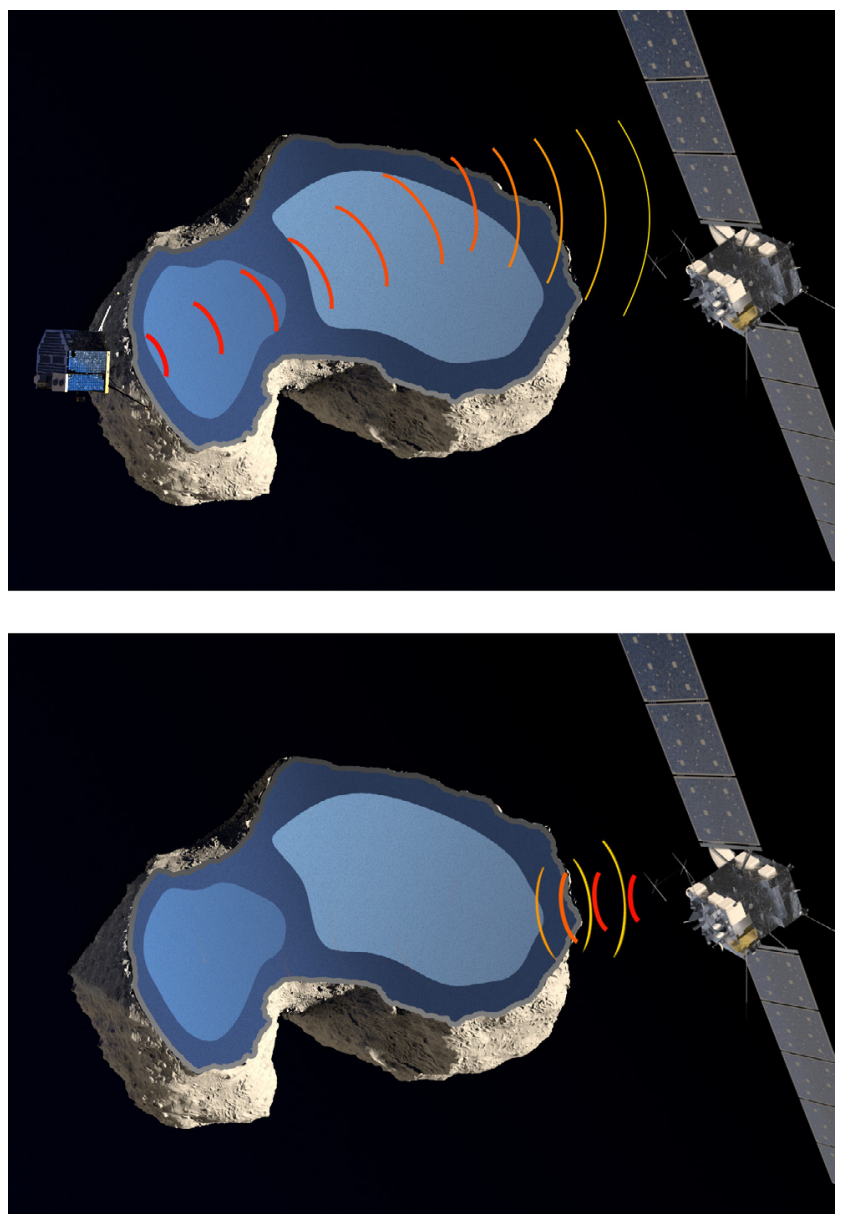

Fig. 3. Bistatic (top) and monostatic (bottom) radar configuration, Artist view from CONSERT/Rosetta (credit: CGI/Rémy Rogez; shape model: Mattias Malmer CC BY SA 3.0, Image source: ESA/Rosetta/NAVCAM, ESA/Rosetta/OSIRIS/MPS/UPD/LAM/IAA/SSO/INTA/UPM/DASP/ IDA).

face images by the Hayabusa spacecraft, it is believed that the asteroid Itokawa is best modeled as an object consisting of a few large fragments covered with a primary layer of large blocks and a secondary layer of fine regolith (Barnouin-Jha et al., 2008 and Fig. 1). Radar is the most mature technique capable of characterizing the internal structure and heterogeneity of an asteroid from submeter to global scale.

Radar sounding does not allow direct 3D-mapping of material mineralogy or composition as is the case for any geophysical remote sensing method. It accesses only the dielectric permittivity: this quantity is related to the properties and the fraction of the different constitutive materials including porosity. The observed permittivity can be compared to the permittivity of "asteroid models" built from laboratory measurement, surface observation and theoretical modeling. The permittivity is then an additional constraint to model internal composition (see for example Herique et al., 2016; Mouginot et al., 2010).

The capability of radar investigations to achieve the mission science objectives is determined mainly by the choice of the frequency, bandwidth and power of the transmitted radio signal:

\subsection{Radar specification}

Frequency typically determines penetration depth (Davis and Annan, 1989): for low-loss materials, such as ice or high-porosity regolith, the dielectric properties are essentially independent of frequency. However, for highloss materials, like most dense rocks, penetration is approximately proportional to the wavelength. Radar propagation in volumetrically heterogeneous media (consisting of variations in permittivity, as well as the size and geometry of blocks and voids) is also determined by the size of heterogeneities with respect to the wavelength $(\lambda)$ : radar waves propagate coherently in a medium with a typical constituent size that is significantly larger than $\lambda$, although it can be refracted at any interface. Radar waves also propagate coherently through media where the heterogeneities are much smaller than $\lambda$; under these conditions, radar waves propagate as if they were in a perfectly homogeneous media. When the radar waves encounter heterogeneities whose size is of the same order of magnitude as $\lambda$, they result in scattering and the loss of wave coherence. For an aggregate, this effect depends on both the size of the fragments and the size of the voids between them. Stated another way, for the same-size block distribution, an increase in macro porosity will increase scattering, while very small values of macro porosity will preserve the wave coherence independently of block size.

Bandwidth determines range resolution (Davis and Annan, 1989). Since it is not possible to transmit a bandwidth that is larger than the highest frequency of the radar, an optimum needs to be defined between the requirements of high penetration, high resolution and the trade off with the technical constraints, especially the antenna size. In other words, deep investigation requires low frequencies in order to reduce dielectric and scattering losses and is then resolution-limited; while regolith sounding requires high bandwidth in order to image structures with higher resolution and is then penetration-limited. Then we have to consider two different frequency ranges to achieve our scientific goals for deep interior and regolith characterization. From a technical point of view, this means two separate radar instruments or one instrument with two channels sharing some digital electronics and ground segment but with separated analog electronics and antenna sets.

\subsection{Radar concept}

This frequency/bandwidth trade-off has to be replayed for each target or mission taking into account the stateof-knowledge for a given asteroid in terms of structure (scattering) and composition (dielectric losses) and also the mission profile, its constraints and opportunities: inorbit duration, orbit altitude, instrument accommodation constraints in term of mass, data volume and antenna size, 
presence of a lander, etc. For this trade-off, two types of radar sounding investigations can be envisaged (Fig. 3): a monostatic radar and a bistatic one.

Monostatic radar sounding is the classical Synthetic Aperture Radar (SAR) configuration where the radar waves are transmitted and their reflected signal received by the Orbiter. At high frequency for surface and near surface characterization, this technique is well-known for both Earth and Planetary observation especially with the SAR onboard Magellan (NASA) and CASSINI (NASA) and MiniSAR on Chandrayaan1 (ISRO). At lower frequency and deeper penetration, there are the Martian radars MARSIS onboard Mars Express (ESA) and SHARAD onboard MRO (NASA); the lunar radar LRS onboard Kaguya (JAXA) and the Jovian moons radars RIME onboard JUICE (ESA) and REASON onboard JEO (NASA). For this technique, part of the incident wave is reflected by the asteroid's surface, scattered by the topography, or continues to propagate into the subsurface, where it may reveal internal structures and sound depths directly related to the radar wavelength. Monostatic radar is less constraining for small bodies sounding than for planets or icy moons due to a significantly lower altitude and lower relative velocity with respect to the surface. It can be applied for regolith sounding with higher frequency or deep investigation with lower frequency allowing a partial or a full penetration.

Bistatic radar tomography in transmission is similar to what has been employed by the CONSERT radar on Rosetta, to characterize the internal structure of the cometary nucleus of 67P/Churyumov-Gerasimenko (Kofman et al., 1998, 2004, 2007). This technique utilizes radar waves transmitted by a Lander and received by an Orbiter after propagation throughout the comet (Fig.3). The measurement is repeated for different positions of the Orbiter with respect to the Lander to achieve a global characterization of the body's deep interior. Tomography in transmission is relevant only for small bodies with a size lower than a few kilometers and propagation losses low enough to have the signal propagating throughout the entire body). In this technique, the waves are delayed, attenuated and possibly scattered by the asteroid internal materials and structures. One can note that this bistatic tomography in transmission is far from the bistatic radar technique in radio science (Simpson, 2007), which uses high frequency communication link from spacecraft to Earth to observe planetary or small body surfaces. This last remote sensing technique of near-subsurface is a by-product of radio science, quite close to ground-based radar observation (Simpson, 2007).

For investigations of the near-subsurface (top $\sim 10 \mathrm{~m}$ ), the use of a high-frequency monostatic radar will enable determination of the depth of the regolith, the detection of buried impact craters, the presence of internal layering, fractures, embedded blocks, variations in lithology and the distribution of macro porosity, all of which can provide valuable insights into the geology and geologic evolution of the asteroid. In this respect, operations and information obtained by a monostatic radar are similar to those of a traditional ground penetrating radar.

For deep sounding and global characterization, depending on the size of the target, monostatic investigations require very low frequencies in order to propagate through the entire target body and back, necessitating the use of large antennas. Moreover, conducting monostatic tomography could require a minimum orbital coverage that impacts both the mission duration and telemetry budget. Bistatic radar investigations can use frequencies that are slightly higher, reducing antenna size and simplifying instrument accommodation. It is less ambitious, but also less demanding in terms of operations time and data budget, with a few small sets of measurement sequences. On the other hand, a bistatic radar requires a surface package. Again, this trade-off has to be considered for each mission, with the presence or absence of a surface package and the fact that a monostatic radar is more demanding in terms of mission implementation.

Hereafter, we will consider mainly a high frequency monostatic radar for shallow subsurface and a low frequency bistatic radar for deep interior as developed for the AIDA/AIM project (Michel et al., 2016) and of its lander MASCOT2 (Ulamec and Biele, 2015; Biele et al., 2017). This is also inherited from the FANTINA proposal: FANTINA was Marco Polo's eldest daughter, the name also stands also for "Fathom Asteroids Now: Tomography and Imagery of a NEA". This suite had been designed for the MarcoPolo-R mission (Barucci et al., 2012), to help answer these questions, support sample acquisition and analysis. FANTINA was finally not selected due to mass considerations.

In order to specify the instrument suite and demonstrate its science performance, we will first review the state of knowledge about asteroid radar properties and propose a dielectric model for the different types of asteroids.

\section{Dielectric model of asteroids}

Wave propagation in asteroids and thus their radar response is driven by the complex dielectric permittivity of the materials: the real part of the relative dielectric constant, $\varepsilon_{r}^{\prime}$ is related to the electromagnetic wave velocity while the imaginary part $\varepsilon_{r}^{\prime \prime}$ is related to the wave absorption and is usually represented by the loss tangent, $\tan \delta$ $=\varepsilon_{r}^{\prime \prime} / \varepsilon_{r}^{\prime}$. Definition of the radar's performance requirements and associated scientific return is therefore fundamentally related to a range of expected complex permittivities of constitutive materials. A careful study of dielectric properties is crucial for both instrument definition and data interpretation.

Rocky materials on Earth give a preliminary range of values: $\varepsilon_{\mathrm{r}}$ ' is constrained within a relatively narrow range of values for almost all minerals, and exhibits some correlation with density: the interval $3<\varepsilon_{r}^{\prime}<10$ is typically used 
to represent compact rocks with little porosity. Variability of $\tan \delta$ in natural materials is somewhat greater than the variability of $\varepsilon_{r}^{\prime}$ and generally correlated with the metallic content: the interval $10^{-3}<\tan \delta<10^{-2}$ can be used to represent most dry rocks with limited metal contents.

For asteroids, the lack of data makes the work difficult. The spectral classes of asteroids are defined from visible and IR ground-based observation: Each asteroid class is assumed to have a distinct bulk composition, which would determine its radar response. Some data can be derived from Earth-based radar observation and from meteorite lab measurement. The main part is obtained by modeling.

More than 700 near Earth asteroids have been observed by Arecibo and Goldstone radars at 2.4, 7.2 and $8.6 \mathrm{GHz}$ (https://echo.jpl.nasa.gov/asteroids/PDS.asteroid.radar. history.html; Naidu et al., 2016; Slade et al., 2011). The most prominent results consist of orbit, spin vector and shape (Magri et al., 2007) and allow inference of internal structures, e.g. from diamond shapes or binary systems (Ostro et al., 2006; Fang and Margot, 2012). The scattered power and the depolarization of radar-waves contribute to constraint of the range of values of porosity and roughness (i.e. the heterogeneity at decimeter scale) of the first meters of the regolith. Both power and depolarization are significantly correlated with spectral class (Benner et al., 2008; Magri et al., 2007; Shepard et al., 2015) but they do not seem to constitute an estimator of the dielectric permittivity.

Magri et al. (2007) show that Earth-based radar echoes received from $\mathrm{M}$-type asteroids are significantly more powerful than those from C-type and S-type, which correspond to the metallic versus rocky composition. C-type and Stype are mainly distinguishable by the polarization ratio, SC/OC (Same-sense Circular polarization to Oppositesense Circular polarization ratio): S-types show a higher depolarization, which corresponds to a larger heterogeneity of the first few meters at the radar wavelength scale. This depolarization is produced by interfaces with curvature radius of the order of the wavelength (Benner et al., 2008) and dielectric contrast. In the shallow subsurface, blocks separated with voids - both with size comparable to the wavelength - will induce a larger wave depolarization than smaller grains with voids or than large blocks embedded in sand. It is the same for the surface and layering, where larger blocks will introduce more depolarization than gravels. In other words, the depolarization ratio characterizes disorder at the scale of the wavelength: size distribution of the constitutive blocks and macro porosity (large voids). This geomorphology is directly related to the collisional history and then to the mineralogy which determines their mechanical properties (Flynn et al., 2009). Composition also contributes directly to the characterization of the returned echoes: for a given block size distribution, the dielectric contrast determines the strength of the scattered power while the dielectric losses determine the typical penetration depth and then the contribution of each internal structure.
Spectral asteroid classes are often associated with meteorite types. Nevertheless, the genetic link remains an open discussion for some classes. Measurements of the dielectric properties of meteorites from the literature are compiled in Table 3 (in annex), but the question is how are these samples representative of their meteorite family and furthermore of asteroids? Measurements were done on a limited number of samples. They may not reflect the compositional variability observed within a given meteorite family (Brearley and Jones, 1998). This results in a large variability in their measured permittivities (Table 3). Similar variability is observed for the density over a larger set of meteorites (Britt and Consolmagno, 2003). The L6 class ranges from $\varepsilon_{\mathrm{r}}=7.8$ to 13.9 (Table 3) and two different samples of the Plainview meteorite (H5) give permittivities ranging from 25 to 46 (Fensler et al., 1962). This variability could reflect the original mineralogy but also heterogeneous terrestrial oxidation. Indeed, a large fraction of the meteorites in Table 3 are not falls. Some of them have been found in cold (e.g., Antarctica) or hot (e.g. North West Africa) deserts. They could have experienced significant terrestrial weathering, especially oxidation and leaching (Bland et al., 2006) resulting in a modification of their original mineralogy, leading to a change of the permittivity. Most of the measurements have been performed on raw samples (Table 3) while the others have been done on powdered samples: the permittivity of the material without porosity can be extrapolated using mixing formulae (Herique et al., 2016). Nevertheless, this estimation is biased since the powder state of rocky meteorites reduces significantly both the real permittivity and the loss tangent, as observed by Campbell and Ulrichs (1969).

An alternative approach to determine dielectric properties of meteorites is to use measurements on individual components identified in meteorites and to perform analytical mixing of these components using dielectric mixing formulas (Sihvola, 1999). A compilation of permittivity of minerals and organics from the literature can be found in Herique et al. (2016) and relevant candidates are summarized in Table 4 (in annex). This compilation reveals a large variability for a given material. This may be partially due to the natural variability of minerals but also most likely to the variability of the experimental conditions (frequency range, temperature, laboratory equipment) and sample preparation (desiccation, powder sieving). This variability is significantly larger for the loss tangent, which is known to be extremely sensitive to the residual moisture, and also to be difficult to accurately measure when lower than a few $10^{-2}$ (Stillman and Olhoeft, 2008).

These two approaches ("bulk" measurements vs. "analytical mixing of individual components") were considered for the three primary classes of asteroids from Tholen (1989) in Table 1. In this table, we did not consider asteroid subtypes from which the connection with meteorites and mineralogy is more speculative. 
Table 1

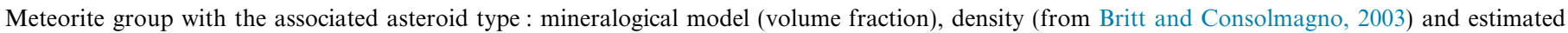
permittivity based on Maxwell Garnet modeling.

\begin{tabular}{|c|c|c|c|c|c|c|c|c|}
\hline Asteroid type & Meteorite group & Silicates & Phyllosilicates & Carbons & Metals & Density $\left(\mathrm{kg} \mathrm{m}^{-3}\right)$ & $\varepsilon$ & $\tan \delta(\max )$ \\
\hline \multirow[t]{3}{*}{$\mathrm{C}$} & $\mathrm{CR}$ & $91.0 \%$ & $0.0 \%$ & $2.0 \%$ & $7.0 \%$ & $3230 \pm 280$ & 8.2 & 0.019 \\
\hline & $\mathrm{CM}$ & $25.0 \%$ & $70.0 \%$ & $5.0 \%$ & $0.0 \%$ & $2710 \pm 110$ & 6.3 & 0.029 \\
\hline & $\mathrm{CI}$ & $10.0 \%$ & $80.0 \%$ & $10.0 \%$ & $0.0 \%$ & $2120 \pm 400$ & 5.9 & 0.037 \\
\hline \multirow[t]{3}{*}{ S } & $\mathrm{H}$ & $91.0 \%$ & $0.0 \%$ & $1.0 \%$ & $8.0 \%$ & $3640 \pm 120$ & 9.3 & 0.012 \\
\hline & $\mathrm{L}$ & $95.0 \%$ & $0.0 \%$ & $1.0 \%$ & $4.0 \%$ & $3510 \pm 110$ & 8.3 & 0.012 \\
\hline & $\mathrm{LL}$ & $97.0 \%$ & $0.0 \%$ & $1.0 \%$ & $2.0 \%$ & $3480 \pm 80$ & 7.8 & 0.012 \\
\hline $\mathrm{M}$ & Mesosiderites & $25.0 \%$ & $0.0 \%$ & $0.0 \%$ & $75.0 \%$ & $4400 \pm 300$ & & \\
\hline
\end{tabular}

\subsection{S-type asteroids}

S-type asteroids are associated with L, LL and $\mathrm{H}$ meteorites' groups (Table 1). Ordinary chondrites are dominated by silicates with a small volume fraction of metal varying from about $2 \%$ for LL group to $8 \%$ for $\mathrm{H}$ group (Table 1). Although $\mathrm{H}$ chondrites dominate the collections of ordinary chondrites, they are suspected to originate from a limited number of parent bodies and cannot be considered as the dominant model for the S-type asteroid population in the main belt (DeMeo and Carry, 2013). The radar properties of the S-group as observed from Earth allow us to clearly distinguish from C- and Mgroups. However Benner et al. (2008) noted the proximity of the radar response of some S-type asteroids with Mtypes and suspected a possible iron-stone composition for some members of this taxon.

For the tested meteorites, the real permittivity ranges from 7 to 20 for LL5, L5 and L6 groups (without porosity) while it can be as high as 81 for H5 (Table 1). The permittivity appears directly correlated to the metal content and composition variability could also explain the difference observed by Fensler et al. (1962) between two samples of the same meteorites (Leedy and Plainview, Table 1). Likewise the loss tangent increases significantly with metal proportion from less than $10^{-2}$ for LL5 to $\sim 3.10^{-2}$ for L5/L6 and up to more than 0.1 for $\mathrm{H} 5$.

Table 4 presents silicates with permittivities ranging from 7.1 to 7.9 for the real part at $1 \mathrm{MHz}$ for olivine, pyroxenes and forsterite. The real permittivity of the dunite is expected to be in the same range after compensation of the porosity with mixing formulas. To model mineral fraction of S-type asteroids, we will consider values ranging from 6.8 to 8 for the real part of the permittivity in a frequency range from $10 \mathrm{MHz}$ to $1 \mathrm{GHz}$. The loss tangent is more difficult to evaluate, with measured values ranging from $8.10^{-4}$ to $4.10^{-2}$. The majority of laboratory measurement was done at room temperature: a significant overestimation due to some residual sample moisture can be suspected. Moreover, asteroid interiors are expected to be significantly colder, while dielectric losses of minerals tend to decrease with decreasing temperature in this $10 \mathrm{MHz}$ to $1 \mathrm{GHz}$ frequency range. Therefore we will consider a maximum loss tangent lower than $10^{-2}$ for the silicate fraction.
The influence of iron/nickel fraction is more difficult to constrain and depends on the chemical states (metallic versus oxides or sulfides), on the topology and impurities content of the metal inclusions. In LL, L and $\mathrm{H}$ meteorites, metals consist mainly of metallic inclusions with millimeter size, far from the percolation threshold as minor components $(<10$ vol. $\%)$. These inclusions increase the overall real permittivity but not the loss tangent, in an unexpected way (Sihvola and Lindell, 1992). This behavior could explain why L6 and L5 permittivities in Table 1 are larger than those measured for silicates (Table 4). It could also explain the large permittivities measured for H5. A more limited part of the iron/nickel content is bound in molecular species without free charge but a large loss tangent; as a minor component they do not have a significant impact on the real part of the permittivity, although the loss tangent increases significantly by an amount that is controlled by the nature of the oxide or sulfides. The organic fraction is minor $(\sim 1 \%)$ and constituted by mature carbon (high $\mathrm{C} /$ $\mathrm{H})$. This can be modeled by graphite, and impacts mainly on the loss tangent. Permittivities' estimation from mineralogy using mixing formulas (Table 1) range from 7.8 for LL to 9.3 for $\mathrm{H}$ class while the loss tangent is close to the loss tangent considered for the silicates. The comparison with measured values shows a good correlation for LL and L5 meteorites, a slight deviation for L6 and drastic deviation for H5. This deviation has to be understood by the composition itself; our modeling is based on some averaged composition (i.e. metal fraction) while the contents can significantly change inside the group and also from the metal inclusion shape, while the impact on the permittivity is strongly dependent on the topology. Considering $\mathrm{L}$ and LL more representative of the S-type population than $\mathrm{H}$ chondrites, we will consider hereafter permittivities ranging from 7 to 10 for this group and maximum loss tangent in the range of $10^{-2}$ without porosity.

\subsection{C-type asteroids}

C-type asteroids are often associated with carbonaceous chondrites. It has to be noted that only the $\mathrm{Ch}$ and $\mathrm{Cgh}$ subtypes have clear matches among meteorite families (Vernazza and Beck, 2016). Also the CV and CR chondrites have been in the past related to K-type asteroids (Bell, 1988; 
Burbine et al., 2001). The permittivities of only one CR2 and one CM meteorite have been measured in the laboratory giving a real permittivity of 2.6 and 3.0 respectively for powdered material with $30 \%$ porosity in the range of $20 \mathrm{MHz}$ to $1 \mathrm{GHz}$ (Table 3, Kofman et al., 2015). As previously noted, measurements on powder lead to an underestimation of both the real permittivity and the loss tangent even after correction for the porosity (Campbell and Ulrichs, 1969). Hence, these measurements have to be considered with some caution. Moreover, the measured CR2 permittivity is lower than the measured CM value despite a significantly larger metal content and a higher density (Table 3, Table 1). This could be explained by terrestrial weathering of the tested sample; NWA 801 (CR2) has indeed experienced moderate to extensive terrestrial weathering with pervasive veins of rust (Connolly et al., 2007). Such oxidation of the metal fraction is expected to reduce significantly the permittivity and could then explain the low permittivity of the NWA 801 (CR2) with regards to NWA $5797(\mathrm{CM})$. Therefore, taking this measurement as representative, would result in significant underestimation of the actual value of the CR2 meteorite class and bias the permittivity assumption for the overall C-type asteroids.

Carbonaceous chondrites (CI, CM, some CV's, CR) show evidence of an aqueous alteration process. In this process, primary minerals (silicates, metals, refractory minerals) reacted with water to produce a more oxidized secondary mineralogy (Brearley, 2006). This process is most intense in the case of CI and CM, where all iron metal was oxidized to iron oxides and a significant fraction of silicates was converted into phyllosilicates. The dielectric properties of $\mathrm{CI}, \mathrm{CM}$ and $\mathrm{CV}$ meteorites were modeled by a ternary mixture, of hydrous silicates (serpentine), anhydrous silicates (olivine) and carbon. Phyllosilicates present lower permittivity from 5 to 7 (porositycorrected) for montmorillonite and serpentine, while the loss tangent seems larger than for the silicates, ranging from $10^{-2}$ to $610^{-2}$. At lower frequency $(\mathrm{kHz})$, Herique et al. (2002) observed that the montmorillonite loss tangent is larger than for dunite, both decreasing drastically when temperature decreases. This higher loss tangent can be explained by mineral hydration and water molecule relaxation. Then we will consider the loss tangent lower than $3 \cdot 10^{-2}$ for phyllosilicates. The organics fraction in carbonaceous chondrites is typically less mature (lower $\mathrm{C} / \mathrm{H}$ ratio) than in $\mathrm{L} / \mathrm{L}$, and is modeled by coals (Table $4-$ we consider here coal with a loss tangent around 0.2 which is the worst case in term of dielectric losses). Estimation of the permittivity gives values from 5.9 to 6.3 for CI and $\mathrm{CM}$, larger than the measured value. The loss tangent is around $3 \cdot 10^{-2}$ mainly driven by phyllosilicates.

The dielectric properties of $\mathrm{CR}$ were modeled in this work by a mixture of anhydrous silicates, low maturity carbon (coal) and a significant fraction of metal (equal proportion of metallic and oxide species). Due to this metal fraction, the estimated permittivity is then 8.2 with a loss tangent around $2 \cdot 10^{-2}$. The real permittivity is signifi- cantly larger than that measured on NWA 801; as explained previously this measurement could not be taken as representative of CR2 due to terrestrial oxidations and to powder state. On the other hand, the modeled value seems consistent with the averaged composition and density of the meteorite family and with the permittivity of the other meteorite families. Therefore we will consider permittivities ranging from 5.9 to 8.2 for C-type associated with a maximum loss tangent $3 \cdot 10^{-2}$.

\subsection{M-type asteroids}

M type asteroids seem to be the vestige of disrupted metallic cores of early planetesimal bodies and require discussion in more detail. A number of asteroid sub-types have been related to metal-rich meteorites: iron meteorites (associated with X-types), pallasite (A-type) or mesosiderite $(\mathrm{Xk})$. This corresponds to pure metal or a metallic matrix embedding rocky inclusions, both explaining the strong radar echoes observed with ground-based radars (Shepard et al., 2015). In Table 3, RKP A79015 is an Antarctica mesosiderite meteorite without significant oxidation or terrestrial weathering. The measured permittivity (Heggy et al., 2012) appears extremely low with regard to its 78\% taenite content (alloy of iron and nickel) and one cannot exclude some significant bias from the tested sample, inducing a large underestimation of the loss tangent.

The radar response of such metallic core material would be dominated by conductivity; with $75 \%$ metallic iron and nickel, macroscopic currents are induced in the matrix at the scale of the wavelength. The penetration depth is then limited by the skin effect at depth, orders of magnitudes lower than the wavelength with some dependence on the impurity contents. Radar echoes are then expected to be powerful and with limited depolarization as observed from Arecibo/Goldstone observations (Shepard et al., 2015).

By comparison to C-type and S-type objects, the echoes from M-type asteroids present a larger standard deviation inside the class, and even for different echoes from the same body. From Earth survey, $40 \%$ of this family present a radar bifurcation (Shepard et al., 2015), a variability of the radar echo power with some areas more close to Ctype or S-type, whereas other parts are fully representative of the $\mathrm{M}$ class. If this variability is not coming from a complex shape or a binary system (Ostro et al., 2000; Shepard et al., 2015), this can be explained by a rocky regolith covering the metallic core with a local silicate enrichment from endogenous or exogenous origin. Studying this regolith, its structure and its origin is the goal of the monostatic high frequency radar to instrument the Heavy Metal mission (M5 Cosmic vision program/ESA) proposed to visit Psyche (Wahlund et al., 2016).

\subsection{Dielectric model}

The present section summarizes our knowledge of the dielectric properties of asteroids and this work has to be 
continued by modeling, as well as laboratory measurement of minerals, meteorites and laboratory-synthesized asteroid analog mixtures, over a large range of temperatures, porosities and frequencies. The following section will focus on rocky asteroids and we will no longer consider the radar sounding of $\mathrm{M}$ class bodies, which are very specific, with expected limited penetration depth. In Fig. 4 the key elements of dielectric model properties versus porosity for Stype (solid lines) and C-type (dashed lines) are summarized. For each type, the two curves delimit the range of variability coming from the variability of the real part of the permittivity without porosity in our modeling above (from 5.9 to 8.2 and from 7 to 10 for C-type and S-type respectively). In this model, we have considered only the maximum value of the loss tangent; one has to keep in mind that the dielectric losses derived from these curves will be the maximum dielectric losses expected for such asteroids.

Finally, this model will be used in the next section to support the radar specification and to estimate the associated scientific return for both bistatic low frequency, and high frequency monostatic, radars.

\section{Global characterization with bistatic radar}

A bistatic radar is capable of probing an asteroid's deep interior in order to discriminate between an aggregate or a monolithic structure and to characterize any heterogeneities: presence or absence of voids, compositional heterogeneity and size distribution of constitutive blocks. The radar signal is acquired after propagation from the Lander to the Orbiter through the asteroid body (Fig. 3, top) and its main parameters of interest are the propagation delay, the wave attenuation and the signal texture. The measurements are repeated over many geometries to follow spatial variation of these parameters; for a probe orbiting a small body, geometry change is generally more from body rotation than from spacecraft orbital motion.

As previously explained, the propagation mode inside the body is dependent on the scattering and therefore on the heterogeneity level at wavelength scales; materials with variation of the permittivity at a scale much smaller than the wavelength $(<\lambda / 10)$ are seen as homogeneous by radar waves. It is the propagation mode inside monolithic rock with some micro-porosity and different minerals; it is also the case for a pile of sand with some macro porosity. Materials with heterogeneity at the scale of the wavelength (between $\lambda / 10$ and 10 $)$ ), like an aggregate of meter-size blocks, produce scattering and so spreading of the radar waves that leads to a typical signature related to the statics of scattered block size. Moreover, larger-scales interfaces $(>10 \lambda)$ correspond to geometric optics with refraction and reflection. It is the propagation behavior for internal layers, blocks embedded in sand, large voids or separated large blocks.

In other words, an aggregate of sand and a monolith correspond to similar wave behavior and then similar signal texture without significant wave spreading; the mean permittivity and its spatial variability as well as possible layers, basal regolith interface or embedded block allow discrimination between the two models. On the other hand, an aggregate of larger blocks produces wave scattering and signal spreading which inform directly on the size of the constitutive blocks and their spatial distribution. In practice, the internal structure could be more complex, as proposed for Itokawa (section II - 1. and Fig. 1) mixing different propagation waves: several acquisitions and tomography processing will then allow retrieval of internal structure.

Hence, the heterogeneity scales compared to the wavelength and the wave propagation inside the body determine the observable parameters present in the signal, and the inversion approaches to be applied.

\subsection{Frequency and bandwidth}

For the in-transmission tomography, the propagation delay provides a direct estimation of the average permittivity along the wave propagation path from the lander to the orbiter (Kofman et al., 2015). This physical quantity can
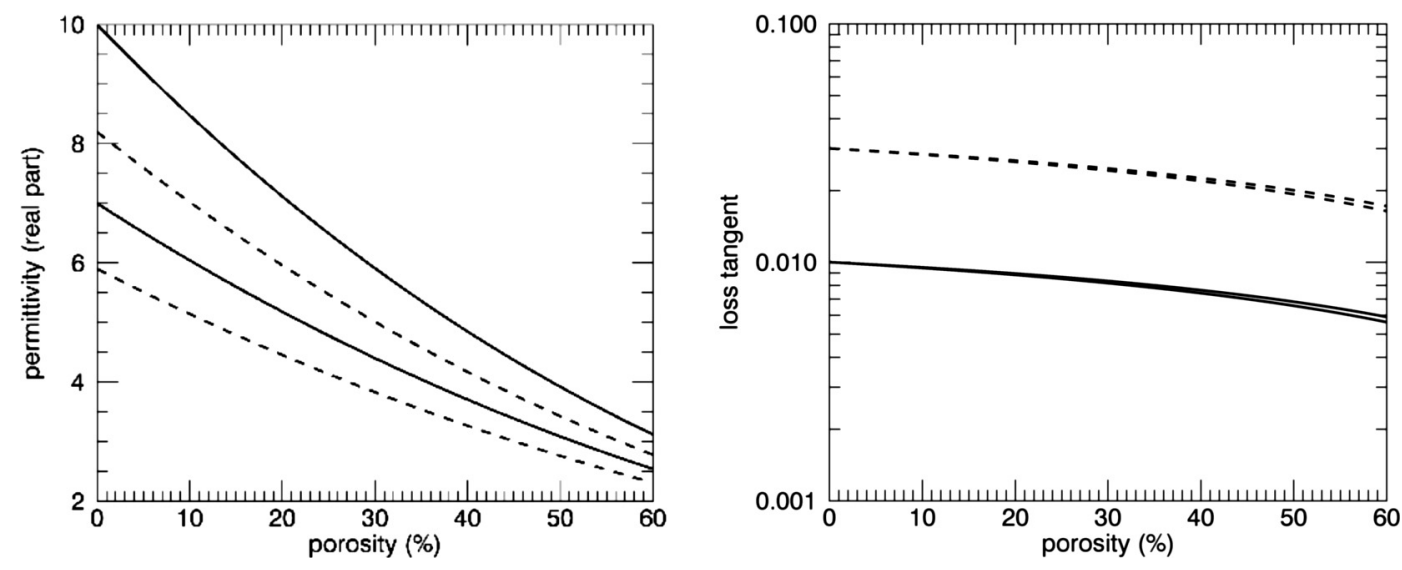

Fig. 4. Real permittivity and maximum loss tangent versus porosity for S-type (solid lines) and C-type asteroids (dashed lines) based on Looyenga mixture formula. Without porosity, S-type asteroid permittivity ranges from 7.0 to 10.0 with a maximum loss tangent equal 0.01 and C-type asteroid permittivity ranges from 5.9 to 8.2 with a maximum loss tangent of 0.03 . 
then be retrieved in terms of composition (Herique et al., 2016). We consider the AIDA/AIM scenario with the MASCOT2 lander placed on the surface of the secondary of Didymos, an S-type binary asteroid, whose moon diameter is around $160 \mathrm{~m}$ (Michel et al., 2016). Fig. 5 (left) shows the variation of the optical length (equivalent to the propagation delay) versus the porosity for S-type permittivities as modeled (section IV - 1. and Table 1). To detect a variation of $5 \%$ in the mean porosity along a propagation path of $160 \mathrm{~m}$ length, a resolution better than $15 \mathrm{~m}$ on the optical length is required. This is equivalent to a time resolution of about $50 \mathrm{~ns}$ and a bandwidth of 20 MHz. This corresponds to an accuracy better than $\Delta \varepsilon=$ 0.6 for permittivity averaged along the propagation pass. For a larger body like the main body of this system, whose diameter is around $800 \mathrm{~m}$, a resolution of $60 \mathrm{~m}$ would be sufficient ( $\mathrm{RF}$ bandwidth $\mathrm{BW}=5 \mathrm{MHz}$ ).

The carrier frequency has to be determined from a trade-off between the bandwidth requirement, the antenna design and accommodation (size and matching), the electronic design (dispersion level) and signal to noise ratio performance (propagation losses and galactic noise). Fig. 5 (right) shows how the dielectric losses increase with the frequency for this moonlet: the attenuation is equal to $0.091 f \sqrt{\varepsilon} \tan \delta d$ in $\mathrm{dB}$ where $f$ is the operating frequency $(\mathrm{MHz})$ and $d$ is the path-distance within the asteroid (m). Up to $100 \mathrm{MHz}$, the radio noise is dominated by the galactic background (Fig. 6, Kraus, 1986) which is significantly stronger than the antenna and receiver thermal noise and drives the radar sensitivity as well as radar receiver dynamics. The dielectric losses increase with frequency while at the same time, the galactic noise decreases (Fig. 6). 60 $\mathrm{MHz}$ ( $\lambda=5 \mathrm{~m}$ in vacuum) appears to be a good compromise considering antenna accommodation constraints and target size. With dielectric losses lower than $20 \mathrm{~dB}$ for the S-type model and $40 \mathrm{dBm}$ transmitted power (Table 2), a $10 \mathrm{~km}$-radius orbit offers a comfortable signal to noise ratio (SNR) after processing $(60 \mathrm{~dB})$ for the moon $(160 \mathrm{~m}$ diameter). This signal is still larger than the noise for the main body, but with a more limited margin $(\mathrm{SNR}>5$ $\mathrm{dB})$. It is more difficult to estimate attenuation due to scattering, which becomes significant when the scatterers are numerous at a dimension comparable to the wavelength $(\lambda)$, so attenuation itself provides information about internal structure.

The design of the whole instrument is driven by the accuracy requirement of propagation delay which defines the in-time transponder structure associated with accurate local oscillators as described in several papers (Kofman et al., 1998, 2004, 2007). Dedicated signal pre-processing to resynchronize the transponder allows significantly improved propagation delay accuracy (a factor of 10) with regards to the bandwidth in case of favorable SNR (Pasquero et al., 2017). The accuracy limitation is then

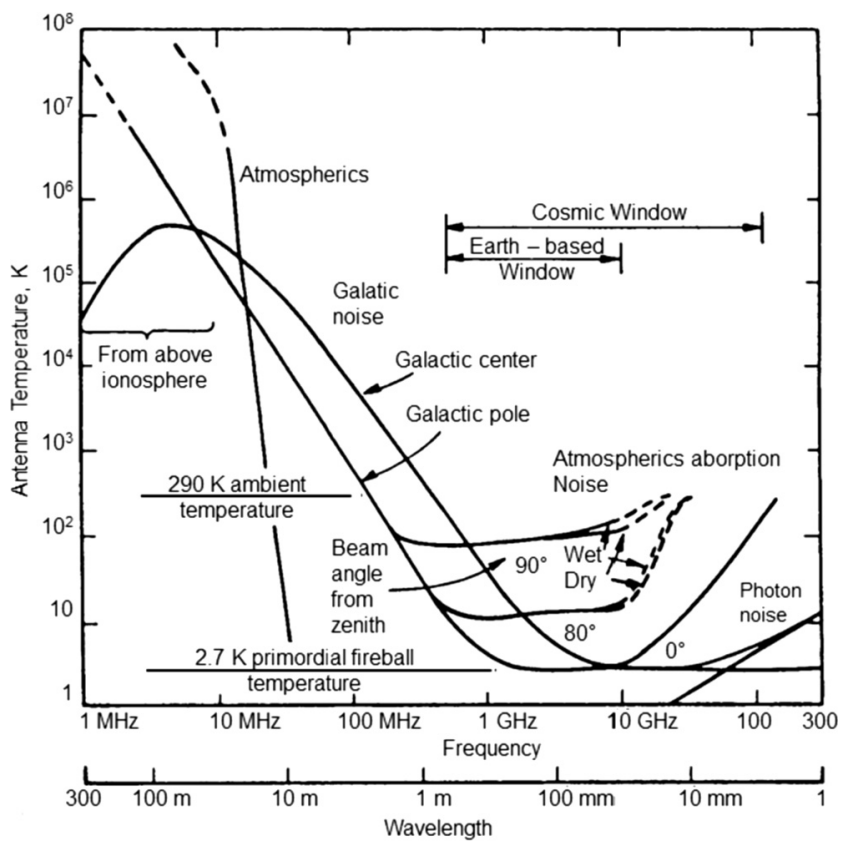

Fig. 6. Galactic Noise: Antenna sky temperature as a function of the frequency and zenith angle (reworked from Kraus, 1986).
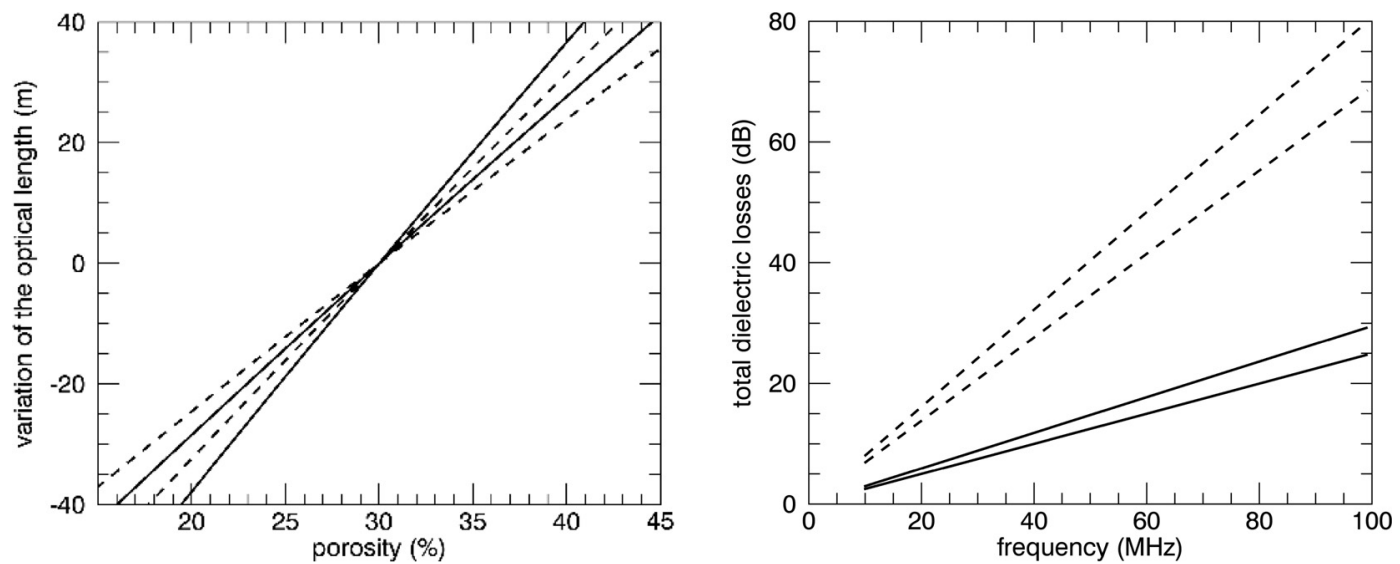

Fig. 5. Bistatic radar sensitivity for the $160 \mathrm{~m}$ moon of the Didymos system. Variation of the optical length versus porosity (left) and total dielectric losses after $160 \mathrm{~m}$ propagation inside the body with $40 \%$ porosity (right). S-type asteroid permittivity (solid lines) ranges from 7.0 to 10.0 with a maximum loss tangent equal to 0.01 . C-type asteroid permittivity (dashed lines) range from 5.9 to 8.2 with a maximum loss tangent equal to 0.03 . 
Table 2

Main characteristics and performance of the bistatic radar and high frequency monostatic radar for the AIDA/AIM mission.

\begin{tabular}{|c|c|c|c|}
\hline & \multicolumn{2}{|l|}{ Bistatic Radar } & \multirow[t]{2}{*}{ Monostatic Radar } \\
\hline & Orbiter & Lander & \\
\hline Frequency: nominal & \multicolumn{2}{|c|}{$50-70 \mathrm{MHz}$} & $300-800 \mathrm{MHz}$ \\
\hline Frequency: extended & \multicolumn{2}{|c|}{$45-75 \mathrm{MHz}$} & $\mathrm{Up}$ to $3 \mathrm{GHz}$ \\
\hline Signal & \multicolumn{2}{|c|}{ BPSK } & Step frequency \\
\hline Resolution & \multicolumn{2}{|c|}{$10-15 \mathrm{~m}(1 \mathrm{D})$} & $1 \mathrm{~m}(3 \mathrm{D})$ \\
\hline Polarization & Circular (AIM) & Linear (Mascot) & $\begin{array}{l}\text { Tx: } 1 \text { Circular } \\
\text { Rx: OC and SC }\end{array}$ \\
\hline Tx power & \multicolumn{2}{|c|}{$12 \mathrm{~W}$} & $20 \mathrm{~W}$ \\
\hline Pulse repetition & \multicolumn{2}{|c|}{$5 \mathrm{~s}$} & $1 \mathrm{~s}$ (typical) \\
\hline Sensitivity & \multicolumn{2}{|c|}{ Dynamics $=180 \mathrm{~dB}$} & $\mathrm{NE} \sigma 0=-40 \mathrm{~dB} \mathrm{~m}{ }^{2} / \mathrm{m}^{2}$ \\
\hline Mass & & & \\
\hline Electronics & $920 \mathrm{~g}$ & $920 \mathrm{~g}$ & $830 \mathrm{~g}$ \\
\hline Antenna & $470 \mathrm{~g}$ & $230+100 \mathrm{~g}$ & $1560 \mathrm{~g}$ \\
\hline Total w/o margin & $1390 \mathrm{~g}$ & $1250 \mathrm{~g}$ & $2390 \mathrm{~g}$ \\
\hline Power max/mean & $50 \mathrm{~W} / 10 \mathrm{~W}$ & $50 \mathrm{~W} / 10 \mathrm{~W}$ & $137 \mathrm{~W} / 90 \mathrm{~W}$ \\
\hline Typical Data (Gbit) & 1 & 0.3 & 300 Gbit \\
\hline
\end{tabular}

given by the orbit restitution accuracy (Herique et al., 2015). The main characteristics and performance of the bistatic radar developed for the AIDA/AIM mission are summarized in Table 2.

\subsection{Deep interior}

The first observable parameter deduced from the radar signal is the real part of the mean permittivity of the body. It is derived from the group delay of the main path, introduced when the asteroid is inserted into the propagation path. The second is the dielectric loss tangent derived from the mean absorption of radio waves as the signal propagates through the body. When combined with other measurements like density estimation from radio science, the dielectric permittivity allows an estimation of the composition and porosity of the bulk material of the asteroid (for CONSERT see Kofman et al., 2015; Herique et al., 2016). With observations along an arc of an orbit, the mean permittivity averaged along the propagation path can be calculated for different positions of the orbiter. Its variation as a function of the orbital position is related to large scale heterogeneities inside the body: gradient of density, presence of large boulders, compositional heterogeneity between large fragments, and variation of the size distribution in an aggregate, variation of the composition or micro-porosity within a monolithic structure. Such kinds of internal structure will also induce the presence of multiple propagation paths received at the same orbiter position.

The statistical characterization of the spatial variation of the signal is a way to retrieve the typical size and contrast of the heterogeneity (Herique et al., 1999); this approach provides robustness in the case of limited spatial coverage of the observation (i.e. an orbit or an arc of orbit). Full tomography of the interior, however, requires better coverage (Eyraud et al., 2018; Barriot et al., 1999) and is significantly improved by multiple source positions at the asteroid surface as provided by lander mobility or from the deployment of several transponders (Pursiainen and Kaasalainen, 2014). The signal texture is related to the scattering properties and also to heterogeneities at scales of the wavelength. A direct measurement of the peak spreading allows the quantification of this heterogeneity in terms of size and contrast (for 67P and CONSERT, see Ciarletti et al., submitted for publication). It is a way to discriminate between an aggregate of large blocks and a monolith. As for the mean permittivity, the spatial variation of signal texture gives statistical properties of the large scale heterogeneities. For large scattering, propagation becomes incoherent and signal power decreases rapidly and only a partial tomography will be possible in chords through the asteroid.

During an arc of an orbit, grazing angle tomography corresponds to a specific geometry tomography when the spacecraft is disappearing behind the horizon with respect to the lander. This geometry allows dedicated inversion approaches to retrieve near surface layering or gradient of permittivity (Ilyushin et al., 2003; Ciarletti et al., 2015). For a binary system, with the lander on the surface of one body, the signal transmitted from the lander in the direction of the second one is weak due to antenna directivity. Depending of the size of the second body, it could be difficult to expect deep sounding of this body; thus grazing angle is then a procedure to provide some information on the near subsurface of this second body. This configuration was considered for AIM mission to probe the Didymos' main body, with MASCOT landed on surface of its moon.

Aggregate or monolithic structure can therefore be discriminated from the mean permittivity and the signal peak enlargement. For a rubble pile asteroid, bistatic tomography will allow us to estimate the size distribution and the internal structure of the individual blocks, possibly by direct imaging in the case of large block sizes and high con- 
trasts, and more generally by statistical analysis of the scattered signal. The estimation of the mean permittivity of the blocks is a way to determine macro- versus micro-porosity in and throughout the regolith, whose spatial variations highlight the heterogeneity of the parent bodies and segregation mechanisms during cratering, disruption, and re-accretion.

In the case of a monolithic asteroid, there is only microporosity, plus the existence of fracture planes from impact and thermal/cooling stress, and possible compositional boundaries. As before, images of the asteroid interior and spatial distributions of the permittivity determine the heterogeneity. A more advanced analysis by statistical or imagery approach will allow the characterization of the heterogeneity inside a monolith, whereas fracture planes and material contrasts would show up as strong reflectors.

\subsection{Gravity field and dynamical state}

The bistatic radar is also a way to have a direct measurement of the lander-to-orbiter distance during visibility periods with an accuracy better than a few meters. During the Rosetta (ESA) mission this mode demonstrated its efficiency in localizing the Philae Lander after its unexpected bounces (Herique et al., 2015). This mode is more than an operational-only mode and can contribute to science too. This secondary objective addresses mainly the determination of the gravitational field and the dynamical state, especially for a binary system like Didymos (Michel et al., 2016).

With a typical asteroid rotational period lower than $12 \mathrm{~h}$ and a spacecraft orbiting at $10 \mathrm{~km}$, the asteroid rotation dominates the variation of the lander/orbiter geometry (for the tomography we consider the asteroid-fixed reference frame and we describe then the orbiter motion in the rotating frame). The lander-to-orbiter distance when the lander is visible to the orbiter is determined by the asteroid gravitational field and by the possible precession or nutation of this body. The gravity field is mainly determined from Doppler tracking of the spacecraft from Earth; to reconstruct trajectories, the direct measurement of the body-to-orbiter distance is additional information with a lower accuracy but a better configuration (direct measurement) and a different line of sight as compared to the Earth-body direction. In the same way, the dynamical state is generally determined from optical data. Radar ranging adds a third direction to the optical image to improve navigation and then determination of the dynamical state.

During the lander descent, radar ranging is also a way to contribute to the estimation of near-field components of the gravitational field. Again, to reconstruct lander trajectory, ranging is fully complementary to optical tracking, offering the third direction.

\section{Near sub-surface imagery with monostatic radar}

The aim of the monostatic radar is to investigate the shallow subsurface of the asteroid down to a few tens of meters depth with meter resolution. Such resolution will allow us to understand the structure of regolith, looking for potential layers and embedded blocks. The radar signal is transmitted by the orbiter and received by it after reflection and scattering by the asteroid surface and near subsurface (Fig. 3, bottom). This signal is acquired as a function of time (i.e. propagation delay) and the measurement is repeated for different observation geometries during an acquisition sequence (i.e. along track motion inducing Doppler) depending on both body rotation and spacecraft motion. Then, the information on the propagation delay and phase (i.e. Doppler) of the received signal over different geometries allows calculation of near-surface scattering properties from the signal amplitude by Synthetic Aperture Radar (SAR) processing. Both the instrument design and the processing are determined by the geometry of small body observation.

\subsection{Synthetic aperture radar for small bodies}

SAR geometry for asteroids deviates drastically from spaceborne stripmap SAR as is used for Earth or planetary observations: The along-track motion is generally dominated by the rotation of the asteroid, given a relative motion lower than meters per second, which relaxes constraints on the pulse repetition frequency (PRF). The spacecraft could be significantly closer to the asteroid; typically at $10 \mathrm{~km}$ range and down to few hundred meters for the AIDA/ AIM mission (or less for a sample return mission) which improves the link budget but limits transmission duration (Tx duration $<$ round-trip propagation delay).

At this distance, when the antenna size is limited and its pattern (i.e. field of view) covers the entire small-body, the

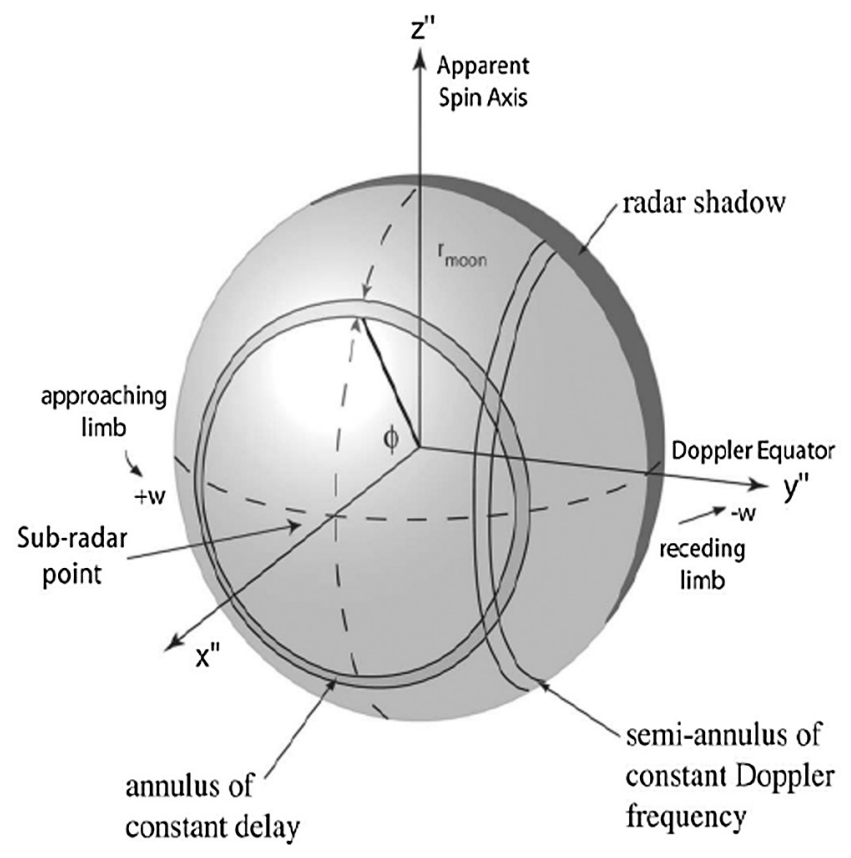

Fig. 7. Geometry of acquisition. 
returned signal is limited by the extension of the body and not by the antenna pattern ("target limited" and not "beam limited", Fig. 7). Therefore, radar requirements and performance are driven by the geometry and not by the pattern. Due to the asteroid's small dimension, range and Doppler are not separable and full-SAR processing including range compensation is required, as for Ultra-Wide Band (UWB) radar (Ulander and Frolind, 1998). In this geometry, the performance will vary with the latitude and the longitude on the asteroid surface (resolution, sensitivity, ambiguity ratios, etc.), as well as the incidence of observation. A signal return from the whole target induces north/south ambiguities: a point at the surface is aliased symmetrically with the ground track (Fig. 7) with some SAR defocusing when the spacecraft is not in the equator plane (high resolution reinforces this defocus effect, helping to resolve ambiguities).

Multipass acquisition and processing are required to fully resolve this ambiguity and provide $3 \mathrm{D}$ tomography: the propagation delay (RF signal bandwidth) gives the resolution in the line-of-sight dimension; along-track motion due to body rotation gives the resolution in the body's equatorial plane; resolving the third direction requires multiple acquisition sequences with the spacecraft moved in a direction parallel to the asteroid spin axis. Simulations show that 10 acquisition sequences provide a meter resolution in 3D with a dynamic range better than $20 \mathrm{~dB}$ (ambiguity ratio $>20 \mathrm{~dB}$ ).

To achieve our scientific goal the instrument requires a large degree of versatility to be in a position to operate at larger or reduced distance, to be optimized for performance for different latitudes or science objectives. The step frequency concept is the most relevant to offer this versatility by instrument setting. This consists of repetition of the transmission and the reception of a continuous wave with a variable frequency covering step-by-step the desired bandwidth. This repetition is possible due to the relaxed constraints on the PRF and offers the possibility of selecting the bandwidth, the number of frequencies and the transmission duration in order to match with the geometry or to focus investigation of some limited areas (increased performance). The succession of transmission and reception slots in time sharing (i.e. no transmission when receiving) is required to transmit power $(20 \mathrm{~W}-$ Table 2$)$ in order to operate at a large distance $(10 \mathrm{~km})$ but could limit the minimum distance for operations $(>200 \mathrm{~m})$.

Our step frequency radar must operate at frequencies significantly higher than the bistatic radar. This results from a trade-off between the dielectric losses and the resolution; dielectric losses increase with frequency and then limit the penetration depth (Fig. 8) while a large bandwidth - to achieve high resolution - is easiest to be implemented at high frequencies. A nominal bandwidth ranging from 300 to $800 \mathrm{MHz}$ seems to be a good compromise allowing high resolution, better than one meter in range close to the surface (the resolution decreases with depth since the absorption varies with frequency). In terms of polarization, transmitting one circular polarization and receiving the

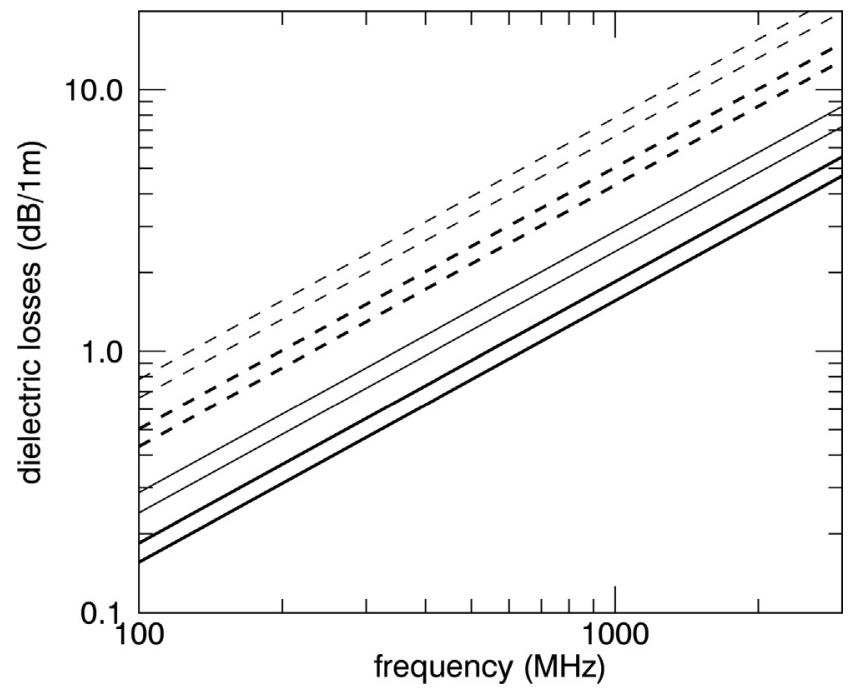

Fig. 8. Monostatic radar sensitivity: total dielectric losses in $\mathrm{dB}$ per meter with $40 \%$ porosity S-type asteroid permittivity (solid lines) ranges from 7.0 to 10.0 with a maximum loss tangent equal to 0.01 . C-type asteroid permittivity (dashed lines) ranges from 5.9 to 8.2 with a maximum loss tangent equal to 0.03 .

two circular polarizations (Same Circular polarization SC and Opposite Circular polarization OC) as classical for earth-based and space-borne planetary SAR is the scenario that avoids complex spacecraft attitude control. The main characteristics and performance of the monostatic radar developed for the AIDA/AIM mission are summarized in Table 2.

\subsection{Regolith and shape model}

As explained earlier, the final resolution and sensitivity will depend strongly on the geometry diversity provided by the observation scenario. The first observed parameter is the backscattering coefficient which quantifies the returned power per surface or volume unit. It is related to the degree of heterogeneity at the scale of the wavelength and to the dielectric contrast of heterogeneities. To illustrate, sand will give lower scattering than decametric blocks for our bandwidth, and the spectral signature could provide relevant information to retrieve block size distribution. The second parameter is the polarization ratio of the scattered wave (SC/OC power ratio) which is sensitive to the multiple scattering and then to the wave penetration.

With one sequence of observation, 2D images mix surface and subsurface contributions, mixing signals arriving with the same delay and Doppler. Such images allow detection of embedded structures masked by surface deposits, which make optical identification impossible. The polarization ratio is then the quantity of interest as currently used for planetary surfaces (see for example Nozette et al., 2010; Thompson et al., 2011 for Mini-RF on LRO and MiniSAR on Chandrayan-1). These images allow identification of not only large blocks or lakes, but also variation of the regolith 
texture and composition in order to understand stratigraphic relations of the different units, layers or lenses. Larger coverage with at least ten sequences of acquisition in relevant configuration will give access to the full subsurface imagery.

Any radar primarily measures the antenna-to-target range, and altimetry data are already present in SAR imagery as long as the radar field of view is covering the full asteroid. With a resolution of up to $30 \mathrm{~cm}$, this altimetry is relevant to support operations that complement on board optical navigation (distance to the closest asteroid point calculated in real-time) or on ground navigation (orbit restitution). It is also of main interest to support determination of the gravity field and of the dynamical state, as explained for the bistatic radar case in section V-3.

SAR processing can also provide the shape model of the asteroid. It is in fact a sub-product of the multipass processing for 3D tomography: the phase difference between multiple acquisitions in different geometries is then used to rebuild the shape by interferometry as done for Venus (Jurgens et al., 1980). On board Rosetta such an instrument would have allowed construction of a shape model of the shadowed part of the nucleus from the early phase of the mission around 67P. For small-body missions, a shape model is derived from optical data: optical and radar measurements are complementary in the geometry point of view but are not sensitive to the same physical quantities. The promising synergy between the two constitutes a wide but relatively unexplored discipline. More classical, multipass approaches are ways to study temporal evolution of surfaces, as is well known for Earth surveys: interferometry to follow shape deformation, coherence to follow disruption, etc. (Goldstein et al., 1988). In the frame of AIDA with its DART component (Cheng et al., 2016), this approach would be implemented to better model the mechanics of the impact.

To end this review, we can consider the interest in operating at higher frequencies. Higher frequencies provide a lower penetration and are therefore less relevant for investigation of regolith. Nevertheless, frequencies up to $2.5 \mathrm{GHz}$ provide greater versatility, and increased altimetry resolution.

They also open the door to promising synergies with ground-based radar observations from Earth. In-orbit acquisition of radar echoes in the same frequency range with an increased resolution could benefit data retrieval of Arecibo observations.

\section{Conclusion}

After many missions, our knowledge of the internal structure of asteroids remains largely unknown and suffers from the lack of any direct observation. The structure of the deep interior and of the regolith is crucial to better understanding and modeling of planetary accretion and collisional history, as well as methods to deflect or disrupt potentially hazardous near-Earth objects. This requires the characterization of the aggregate structure and constitutive block size distribution to model mechanical and thermal properties as well as dynamical behavior. A direct observation of deep interior and regolith is a way also to constrain internal composition and porosity. These measurements are then key to planning any spacecraft interaction with an asteroid in the frame of a sample return mission, a well-defined planetary defense program, or any attempt at asteroid mining.

Radar is certainly the most mature method suitable of characterizing the interior of a small airless body from sub-meter to global scale. Two complementary radars are needed to get the whole information: a bistatic radar around $60 \mathrm{MHz}$ to provide the tomography of the deep interior and a high frequency monostatic radar to characterize the regolith structure.

Bistatic radar allows discrimination between aggregate and monolith from the propagation delay and the signal spreading. Depending on the sounded structure, it gives access to the typical size of the constitutive blocks or heterogeneities and offers new constraints to model composition and porosity. To achieve these goals, the radar resolution has to be better than $7 \mathrm{~m}$ (in vacuum) with multiple sequences of acquisition $(>5)$. The line of sight ranging from lander to orbiter during descent or after landing is to support the determination of the gravity field and the determination of the dynamical state in synergy with other instruments, especially camera.

Monostatic radar operating in a frequency range of 300 $800 \mathrm{MHz}$ provides a $2 \mathrm{D}$ or 3D map of the first tens of meters of the regolith, imaging spatial variability, layers or embedded blocks and allows linking of surface to deep interior properties. This geological map is crucial for any sample return mission (sampling site selection and contextualization) as well as to prepare any asteroid deflection or asteroid mining program. To achieve these goals, the resolution has to be better than one meter in $3 \mathrm{D}$, which requires a dozen sequences of operation. Altimetry data can be directly derived from radar sounding to support estimation of the gravity field and determination of the dynamical state. These data can also contribute to the shape modeling.

For the two instruments, these secondary objectives are more speculative, but promising for science return and operational purposes.

To achieve these science objectives, a bistatic radar and a monostatic radar have been developed with heritage from CONSERT on Rosetta and WISDOM on ExoMars respectively. After the AIDA/AIM phase A/B1, these instruments have been included in several M5 Cosmic Vision proposals for asteroids. After the preliminary selection three smallbody missions carrying the high frequency radar are still in the running: Castalia to Main-belt Comet 133P/ElstPizarro (Snodgrass et al., 2018), Heavy Metal to Psyche (Wahlund et al., 2016) and also DePhine to Deimos (Oberst et al., 2018). We believe that this unique combination of two complementary radars for future asteroid missions will answer the questions that are crucial for science, exploration, planetary defense and in situ resource utilization. 


\section{Acknowledgement}

The radars' development has been supported by CNES's R\&T program ("CONSERT Next Generation" study) and by ESA's General Studies Program (AIM Phase A).

The High Frequency Radar is inherited from WISDOM/Exomars funded by CNES and DLR.

The Low Frequency Radar is inherited from CONSERT/Rosetta funded by CNES and DLR.
Authors thanks Paul Abell and the other reviewer for very useful comments and suggestions which help us to improve significantly our paper.

\section{Appendix A}

See Tables 3 and 4.

Table 3

Dielectric permittivity of meteoritic samples from the literature (modified from Herique et al., 2016).

\begin{tabular}{|c|c|c|c|c|c|c|c|}
\hline Classification & Name & Fall & $\varepsilon$ & $\tan \delta$ & Porosity & Frequency (MHz) & Reference \\
\hline \multirow[t]{2}{*}{$\mathrm{CR} 2$} & NWA 801 & $\mathrm{~N}$ & 2.6 & - & 0.3 & $20-1000$ & Kofman et al. (2015) \\
\hline & & & $3.5<.<4.0$ & - & Compensated & & Herique et al. (2016) \\
\hline \multirow[t]{2}{*}{$\mathrm{CM}$} & NWA 5797 & $\mathrm{~N}$ & 2.9 & - & 0.3 & $20-1000$ & Kofman et al. (2015) \\
\hline & & & $4.0-4.7$ & - & Compensated & & Herique et al. (2016) \\
\hline \multirow[t]{2}{*}{ LL5 } & MAC 88122 & $\mathrm{~N}$ & 4.7 & 4. $10^{-3}$ & 0.3 & $20-1000$ & Heggy et al. (2012) \\
\hline & & & $6.9<.<12.2$ & & Compensated & & Herique et al. (2016) \\
\hline \multirow[t]{2}{*}{ L5 } & MET 01260 & $\mathrm{~N}$ & 5.6 & 4. $10^{-3}$ & 0.3 & $20-1000$ & Heggy et al. (2012) \\
\hline & & & $8.4<.<20.4$ & & Compensated & & Herique et al. (2016) \\
\hline \multirow[t]{5}{*}{ L6 } & Holbrook & $\mathrm{Y}$ & 7.8 & $1.510^{-2}$ & Solid & 450 & Campbell and Ulrichs (1969) \\
\hline & Burderheim & $\mathrm{Y}$ & $9.0-11.9$ & 4. $10^{-2}$ & Solid & 450 & Campbell and Ulrichs (1969) \\
\hline & Colby & $\mathrm{N}$ & $10.6-11.8$ & 5. $10^{-2}$ & Solid & 450 & Campbell and Ulrichs (1969) \\
\hline & Leedy (sample 1) & $\mathrm{Y}$ & $10.4-11.1$ & $3-6.10^{-2}$ & Solid & $420-1800$ & Fensler et al. (1962) \\
\hline & Leedy (sample 2) & $\mathrm{Y}$ & $11.2-13.9$ & $3-4 \cdot 10^{-2}$ & Solid & $420-1800$ & Fensler et al. (1962) \\
\hline \multirow[t]{6}{*}{ H5 } & LEW 85320 & $\mathrm{~N}$ & 5.7 & 2. $10^{-2}$ & 0.3 & $20-1000$ & Heggy et al. (2012) \\
\hline & & & $8.6<.<21.4$ & & Compensated & & Herique et al. (2016) \\
\hline & Forest City & $\mathrm{Y}$ & $16-33$ & 0.11 & Solid & 450 & Campbell and Ulrichs (1969) \\
\hline & Plainview (sample 1) & $\mathrm{N}$ & $25.4-30.4$ & $0.1-0.2$ & Solid & $420-1800$ & Fensler et al. (1962) \\
\hline & Plainview (sample 2) & $\mathrm{N}$ & $32.1-45.9$ & $0.1-0.2$ & Solid & $420-1800$ & Fensler et al. (1962) \\
\hline & Bonita Springs & $\mathrm{N}$ & $43-81$ & $0.13-0.19$ & Solid & 450 & Campbell and Ulrichs (1969) \\
\hline EH4 & Indarch & $\mathrm{y}$ & $130-150$ & $0.065-0.117$ & Solid & 450 & Campbell and Ulrichs (1969) \\
\hline \multirow[t]{2}{*}{ Mesosiderite } & RKP A79015 & $\mathrm{N}$ & 8 & 3. $10^{-3}$ & 0.3 & $20-1000$ & Heggy et al. (2012) \\
\hline & & & $12.3<$ & & Compensated & , & Herique et al. (2016) \\
\hline
\end{tabular}

- Campbell's measurements present a range of variability coming from the explored frequency range.- Fensler's measurements present the same range coming from the frequency and also the characterization of 3 different samples for each meteorite.

- The other measurements have been done for porous materials and don't present any significant variability frequency. In italic, the range of permittivity given by Hashin-Shtrikman bounds (from Sihvola, 1999) for the solid material, without porosity from Herique et al. (2016): the given range is due to the uncertainties from the mixing formula and not due to any variability of the measured value itself. In other words, the permittivity of the solid material is somewhere between the two bounds.

Table 4

Permittivity of relevant minerals and organics (from the literature; compilation of permittivity in Herique et al., 2016).

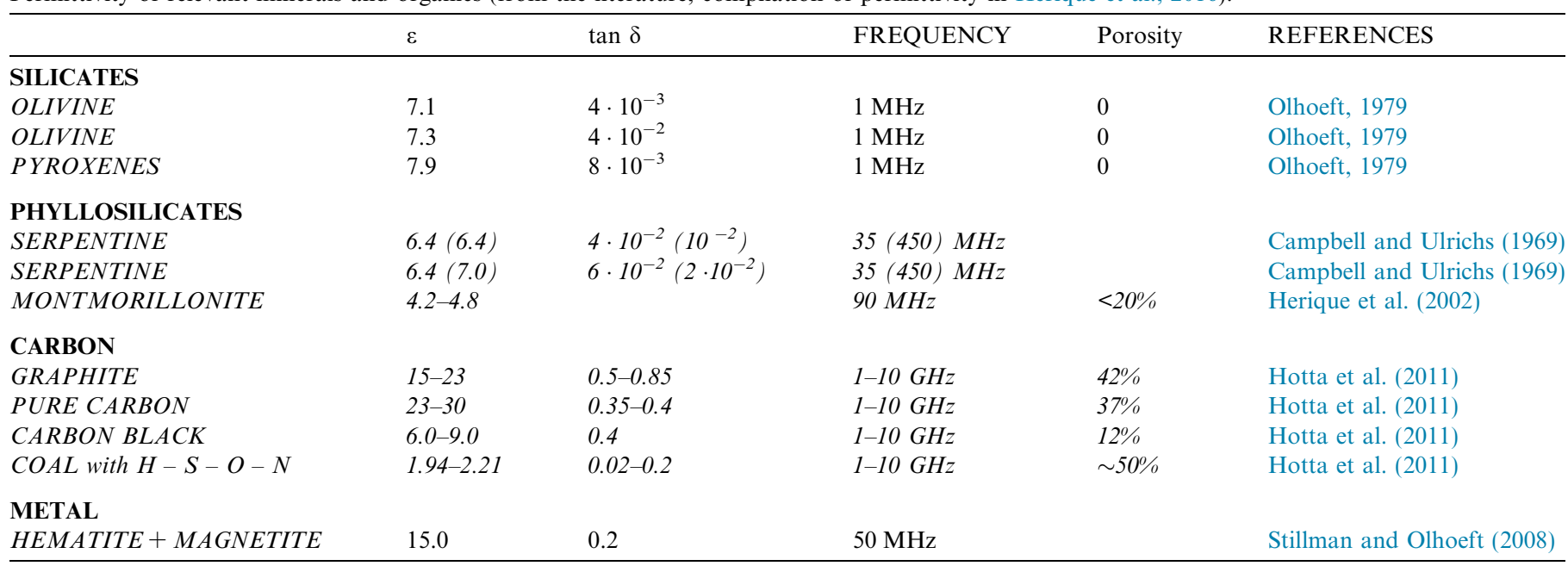




\section{References}

Abe, S., Mukai, T., Hirata, N., Barnouin-Jha, O.S., Cheng, A.F., Demura, H., Gaskell, R.W., Hashimoto, T., Hiraoka, K., Honda, T., Kubota, T., Matsuoka, M., Mizuno, T., Nakamura, R., Scheeres, D.J., Yoshikawa, M., 2006. Mass and local topography measurements of itokawa by hayabusa. Science 312, 1344-1347. https://doi.org/ 10.1126/science.1126272.

Asphaug, E., 2009. Growth and evolution of asteroids. Annu. Rev. Earth Planet. Sci. 37, 413-448. https://doi.org/10.1146/annurev. earth.36.031207.124214.

Asphaug, E., Ryan, E.V., Zuber, M.T., 2002. Asteroid interiors. In: Bottke, W.F., Jr., Cellino, A., Paolicchi, P., Binzel, R.P. (Eds.), Asteroids III. University of Arizona Press, Tucson, pp. 463-484.

Barnouin-Jha, O.S., Cheng, A.F., Mukai, T., Abe, S., Hirata, N., Nakamura, R., Gaskell, R.W., Saito, J., Clark, B.E., 2008. Smallscale topography of 25143 Itokawa from the Hayabusa laser altimeter. Icarus 198, 108-124. https://doi.org/10.1016/j.icarus.2008.05.026.

Barriot, J.-P., Kofman, W., Herique, A., Leblanc, S., Portal, A., 1999. A two dimensional simulation of the CONSERT experiment (Radio tomography of comet Wirtanen). Adv. Space Res. 24, 1127-1138.

Barucci, M.A., Cheng, A.F., Michel, P., Benner, L.A.M., Binzel, R.P., Bland, P.A., Böhnhardt, H., Brucato, J.R., Campo Bagatin, A., Cerroni, P., Dotto, E., Fitzsimmons, A., Franchi, I.A., Green, S.F., Lara, L.-M., Licandro, J., Marty, B., Muinonen, K., Nathues, A., Oberst, J., Rivkin, A.S., Robert, F., Saladino, R., Trigo-Rodriguez, J. M., Ulamec, S., Zolensky, M., 2012. MarcoPolo-R near earth asteroid sample return mission. Exp. Astron. 33, 645-684. https://doi.org/ 10.1007/s10686-011-9231-8.

Barucci, M.A., Dotto, E., Levasseur-Regourd, A.C., 2011. Space missions to small bodies: asteroids and cometary nuclei. Astron. Astrophys. Rev. 19 (48), 1-29. https://doi.org/10.1007/s00159-011-0048-2.

Bell, J.F., 1988. A probable asteroidal parent body for the CO or CV chondrites. Meteoritics 23, 256-257.

Benner, L.A.M., Ostro, S.J., Magri, C., Nolan, M.C., Howell, E.S., Giorgini, J.D., Jurgens, R.F., Margot, J.-L., Taylor, P.A., Busch, M. W., Shepard, M.K., 2008. Near-Earth asteroid surface roughness depends on compositional class. Icarus 198, 294-304. https://doi.org/ 10.1016/j.icarus.2008.06.010.

Benz, W., Asphaug, E., 1999. Catastrophic disruptions revisited. Icarus $142,5-20$

Biele, J., Ulamec, S., Kraus, C., Herique, A., Grygorczuk, J., Lange, C., Grundmann, J.-T., Ho, T-M., Carnelli, I., Galvez, A., Philippe P., Tardivel, S., 2017. MASCOT-2, A lander to characterize the target of an impact asteroid deflection mission, Presentation at 5th IAA Planetary Defense Conference, PDC, Tokyo.

Bland, P.A., Zolensky, M.E., Benedix, G.K., Sephton, M.A., 2006. Weathering of chondritic meteorites. In: Lauretta, D., McSween Jr., H.Y. (Eds.), Meteorites and the Early Solar System II, University of Arizona Press, Tucson, pp 853-867. ISBN: 9780816525621.

Blum, J., Wurm, G., 2008. The growth mechanisms of macroscopic bodies in protoplanetary disks. Annu. Rev. Astron. Astrophys. 46, 21-56.

Brearley, A.J., 2006. The action of water. Meteor. Early Solar Syst. II 943 , 587-624.

Brearley, A.J., Jones, R.H., 1998. Chondritic meteorites. Rev. Mineral. Geochem. 36, 3.1-3.

Britt, D.T., Consolmagno, G., 2003. Stony meteorite porosities and densities: a review of the data through 2001. Meteorit. Planet. Sci. 38, $1161-1180$.

Brownlee, D., Tsou, P., Aleon, J., Alexander, C.M.O., Araki, T., Bajt, S., Baratta, G.A., Bastien, R., Bland, P., Bleuet, P., Borg, J., Bradley, J.P., Brearley, A., Brenker, F., Brennan, S., Bridges, J.C., Browning, N.D., Brucato, J.R., Bullock, E., Burchell, M.J., Busemann, H., Butterworth, A., Chaussidon, M., Cheuvront, A., Chi, M., Cintala, M.J., Clark, B. C., Clemett, S.J., Cody, G., Colangeli, L., Cooper, G., Cordier, P., Daghlian, C., Dai, Z., D'Hendecourt, L., Djouadi, Z., Dominguez, G., Duxbury, T., Dworkin, J.P., Ebel, D.S., Economou, T.E., Fakra, S.,
Fairey, S.A.J., Fallon, S., Ferrini, G., Ferroir, T., Fleckenstein, H., Floss, C., Flynn, G., Franchi, I.A., Fries, M., Gainsforth, Z., Gallien, J.-P., Genge, M., Gilles, M.K., Gillet, P., Gilmour, J., Glavin, D.P., Gounelle, M., Grady, M.M., Graham, G.A., Grant, P.G., Green, S.F., Grossemy, F., Grossman, L., Grossman, J.N., Guan, Y., Hagiya, K., Harvey, R., Heck, P., Herzog, G.F., Hoppe, P., Horz, F., Huth, J., Hutcheon, I.D., Ignatyev, K., Ishii, H., Ito, M., Jacob, D., Jacobsen, C., Jacobsen, S., Jones, S., Joswiak, D., Jurewicz, A., Kearsley, A.T., Keller, L.P., Khodja, H., Kilcoyne, A.L.D., Kissel, J., Krot, A., Langenhorst, F., Lanzirotti, A., Le, L., Leshin, L.A., Leitner, J., Lemelle, L., Leroux, H., Liu, M.-C., Luening, K., Lyon, I., MacPherson, G., Marcus, M.A., Marhas, K., Marty, B., Matrajt, G., McKeegan, K., Meibom, A., Mennella, V., Messenger, K., Messenger, S., Mikouchi, T., Mostefaoui, S., Nakamura, T., Nakano, T., Newville, M., Nittler, L.R., Ohnishi, I., Ohsumi, K., Okudaira, K., Papanastassiou, D.A., Palma, R., Palumbo, M.E., Pepin, R.O., Perkins, D., Perronnet, M., Pianetta, P., Rao, W., Rietmeijer, F.J. M., Robert, F., Rost, D., Rotundi, A., Ryan, R., Sandford, S.A., Schwandt, C.S., See, T.H., Schlutter, D., Sheffield-Parker, J., Simionovici, A., Simon, S., Sitnitsky, I., Snead, C.J., Spencer, M.K., Stadermann, F.J., Steele, A., Stephan, T., Stroud, R., Susini, J., Sutton, S.R., Suzuki, Y., Taheri, M., Taylor, S., Teslich, N., Tomeoka, K., Tomioka, N., Toppani, A., Trigo-Rodriguez, J.M., Troadec, D., Tsuchiyama, A., Tuzzolino, A.J., Tyliszczak, T., Uesugi, K., Velbel, M., Vellenga, J., Vicenzi, E., Vincze, L., Warren, J., Weber, I., Weisberg, M., Westphal, A.J., Wirick, S., Wooden, D., Wopenka, B., Wozniakiewicz, P., Wright, I., Yabuta, H., Yano, H., Young, E.D., Zare, R.N., Zega, T., Ziegler, K., Zimmerman, L., Zinner, E., Zolensky, M., 2006. Comet 81P/Wild 2 under a microscope. Science 314, 1711-1716. https://doi.org/10.1126/science.1135840.

Burbine, T.H., Binzel, R.P., Bus, S.J., Clark, B.E., 2001. K asteroids and CO3/CV3 chondrites. Meteorit. Planet. Sci. 36, 245-253.

Campbell, M.J., Ulrichs, J., 1969. Electrical properties of rocks and their significance for lunar radar observations. J. Geophys. Res. 74, 58675881.

Campins, H., Hargrove, K., Pinilla-Alonso, N., Howell, E.S., Kelley, M. S., Licandro, J., Mothé-Diniz, T., Fernández, Y., Ziffer, J., 2010. Water ice and organics on the surface of the asteroid 24 Themis. Nature 464, 1320-1321. https://doi.org/10.1038/nature09029.

Chambers, J.E., Wetherill, G.W., 1998. Making the terrestrial planets: Nbody integrations of planetary embryos in three dimensions. Icarus 136, 304-327.

Cheng, A.F., 2009. Fundamentally distinct outcomes of asteroid collisional evolution and catastrophic disruption. Planet. Space Sci. 57, 165-172. https://doi.org/10.1016/j.pss.2008.08.013.

Cheng, A.F., 2004. Collisional evolution of the asteroid belt. Icarus 169, 357-372. https://doi.org/10.1016/j.icarus.2004.02.002.

Cheng, A.F., Michel, R., Jutzi, M., Rivkin, A.S., Stickle, A., Barnouin, O., Ernst, C., Atchison, J., Pravec, P., Richardson, D.C., Team, A.I.D. A., Team, A.I.M., 2016. Asteroid impact \& deflection assessment mission: kinetic impactor. Planet. Space Sci. 121, 27-35. https://doi. org/10.1016/j.pss.2015.12.004.

Chesley, S.R., Farnocchia, D., Nolan, M.C., Vokrouhlicky, D., Chodas, P.W., Milani, A., Spoto, F., Rozitis, B., Benner, L.A.M., Bottke, W. F., Busch, M.W., Emery, J.P., Howell, E.S., Lauretta, D.S., Margot, J.-L., Taylor, P.A., 2014. Orbit and bulk density of the OSIRIS-REx target Asteroid (101955) Bennu. Icarus 235, 5-22. https://doi.org/ 10.1016/j.icarus.2014.02.020.

Chesley, S.R., Nolan, M.C., Farnocchia, D., Milani, A., Emery, J., Vokrouhlicky, D., Lauretta, D.S., Taylor, P.A., Benner, L.A.M., Giorgini, J.D., Brozovic, M., Busch, M.W., Margot, J.-L., Howell, E. S., Naidu, S.P., Valsecchi, G.B., Bernardi, F., 2012. The trajectory dynamics of near-earth asteroid 101955 (1999 RQ36). Presented at the Asteroids, Comets, Meteors 2012, p. 6470.

Ciarletti, V., Levasseur-Regourd, A.C., Lasue, J., Statz, C., Plettemeier, D., Herique, A., Rogez, Y., Kofman, W., 2015. CONSERT suggests a change in local properties of 67P/Churyumov-Gerasimenko's nucleus 
at depth. Astron. Astrophys. 583 (A40), 1-7. https://doi.org/10.1051/ 0004-6361/201526337.

Ciarletti et al., 2017. CONSERT Constrains the Internal Structure of 67P at a Few Meter Size Scale, MNRAS (under review).

Connolly, H.C., Smith, C., Benedix, G., Folco, L., Righter, K., Zipfel, J., Yamaguchi, A., Aoudjehane, H.C., 2007. The meteoritical bulletin, No. 92, 2007 September. Meteorit. Planet. Sci. 42, 1647-1694.

Davis, J.L., Annan, A.P., 1989. Ground-penetrating radar for highresolution mapping of soil and rock stratigraphy1. Geophys. Prospect. 37, 531-551.

Delbo, M., dell'Oro, A., Harris, A.W., Mottola, S., Mueller, M., 2007. Thermal inertia of near-Earth asteroids and implications for the magnitude of the Yarkovsky effect. Icarus 190, 236-249. https://doi. org/10.1016/j.icarus.2007.03.007.

Delbo, M., Libourel, G., Wilkerson, J., Murdoch, N., Michel, P., Ramesh, K.T., Ganino, C., Verati, C., Marchi, S., 2014. Thermal fatigue as the origin of regolith on small asteroids. Nature 508, 233. doi:https://doi. org/10.1038/nature13153.

Delbo, M., Mueller, M., Emery, J.P., Rozitis, B., Capria, M.T., 2015. In: Michel, P., DeMeo, F.E., Bottke, W.F. (Eds.), Asteroids IV. Univ. Arizona Press, Tucson, pp 107-128. doi:https://doi.org/10.2458/azu_ uapress_9780816532131-ch006.

DeMeo, F.E., Carry, B., 2013. The taxonomic distribution of asteroids from multi-filter all-sky photometric surveys. Icarus 226, 723-741. https://doi.org/10.1016/j.icarus.2013.06.027.

Durda, D.D., Bottke Jr., W.F., Enke, B.L., Merline, W.J., Asphaug, E., Richardson, D.C., Leinhardt, Z.M., 2004. The formation of asteroid satellites in large impacts: results from numerical simulations. Icarus 167, 382-396. https://doi.org/10.1016/j.icarus.2003.09.017.

Eyraud, C., Herique, A., Geffrin, J.-M., Kofman Eyraud, W., 2018. Imaging the Interior of a Comet from Bistatic Microwave Measurements: Case of a Scale Comet Model. Adv. Space Res. 62, 1977-1986.

Fang, J., Margot, J.-L., 2012. Near-Earth Binaries and Triples: Origin and Evolution of Spin-Orbital Properties. AJ 143, 1-24. doi:https://doi. org/10.1088/0004-6256/143/1/24.

Fensler, W.E., Knott, E.F., Olte, A., Siegel, K.M., 1962. The electromagnetic parameters of selected terrestrial and extraterrestrial rocks and glasses. In: Symposium-International Astronomical Union. Cambridge Univ Press, pp. 545-565.

Flynn, G.J., Durda, D.D., Sandel, L.E., Kreft, J.W., Strait, M.M., 2009. Dust production from the hypervelocity impact disruption of the Murchison hydrous CM2 meteorite: Implications for the disruption of hydrous asteroids and the production of interplanetary dust. Planet. Space Sci. 57, 119-126. https://doi.org/10.1016/j. pss.2008.09.005.

Franchi, I.A., Barucci, M.A., Brucato, J.R., MarcoPolo-M5 Proposal Team, 2017. MarcoPolo-M5 - A Sample Return Mission to a D-Type Near Earth Asteroid Proposed to the ESA Cosmic Vision M5 Call. Presentation at Lunar and Planetary Science Conference XLVIII, The Woodlands, Houston, Texas. 20-24 March, 2017. LPSC Abstract \#2667. <http://www.lpi.usra.edu/meetings/lpsc2017/pdf/2667.pdf>.

Fujiwara, A., Kawaguchi, J., Yeomans, D.K., Abe, M., Mukai, T., Okada, T., Saito, J., Yano, H., Yoshikawa, M., Scheeres, D.J., Barnouin-Jha, O., Cheng, A.F., Demura, H., Gaskell, R.W., Hirata, N., Ikeda, H., Kominato, T., Miyamoto, H., Nakamura, A.M., Nakamura, R., Sasaki, S., Uesugi, K., 2006. The rubble-pile asteroid Itokawa as observed by Hayabusa. Science 312, 1330-1334. https:// doi.org/10.1126/science.1125841.

Garenne, A., Beck, P., Montes-Hernandez, G., Chiriac, R., Toche, F., Quirico, E., Bonal, L., Schmitt, B., 2014. The abundance and stability of "water" in type 1 and 2 carbonaceous chondrites (CI, CM and CR). Geochim. Cosmochim. Acta 137, 93-112. https://doi.org/10.1016/j. gca.2014.03.034.

Goldstein, R.M., Zebker, H.A., Werner, C.L., 1988. Satellite radar interferometry: two-dimensional phase unwrapping. Radio Sci. 23, 713-720. https://doi.org/10.1029/RS023i004p00713.

Heggy, E., Palmer, E.M., Kofman, W., Clifford, S.M., Righter, K., Hérique, A., 2012. Radar properties of comets: parametric dielectric modeling of Comet 67P/Churyumov-Gerasimenko. Icarus 221, 925 939. https://doi.org/10.1016/j.icarus.2012.09.023.

Herique, A., Biele, J., Bousquet, P., Ciarletti, V., Ho, T.M., Issler, J.L., Kofman, W., Michel, P., Plettemeier, D., Puget, P., Souyris, J.C., Ulamec, S., van Zoest, T., Zine, S., 2012. FANTINA: fathom asteroids now: tomography and imagery of a NEA-payload for marco polo $\mathrm{R}$ CV3/ESA mission. Presented at the EGU General Assembly Conference Abstracts, p. 9633.

Herique, A., Gilchrist, J., Kofman, W., Klinger, J., 2002. Dielectric properties of comet analog refractory materials. Planet. Space Sci. 50, $857-863$.

Herique, A., Kofman, W., Beck, P., Bonal, L., Buttarazzi, I., Heggy, E., Lasue, J., Levasseur-Regourd, A.C., Quirico, E., Zine, S., 2016. Cosmochemical implications of CONSERT permittivity characterization of 67P/CG. Mon. Not. R. Astron. Soc. 462, S516-S532. https:// doi.org/10.1093/mnras/stx040.

Herique, A., Kofman, W., Hagfors, T., Caudal, G., Ayanides, J.-P., 1999. A characterization of a comet nucleus interior: inversion of simulated radio frequency data. Planet. Space Sci. 47, 885-904.

Herique, A., Rogez, Y., Pasquero, O.P., Zine, S., Puget, P., Kofman, W., 2015. Philae localization from CONSERT/Rosetta measurement. Planet. Space Sci. 117, 475-484. https://doi.org/10.1016/j. pss.2015.08.020.

Hotta, M., Hayashi, M., Lanagan, M.T., Agrawal, D.K., Nagata, K., 2011. Complex permittivity of graphite, carbon black and coal powders in the ranges of X-band frequencies (8.2 to $12.4 \mathrm{GHz})$ and between 1 and $10 \mathrm{GHz}$. ISIJ Int. 51, 1766-1772. https://doi.org/ 10.2355/isijinternational.51.1766.

Horstmann, M., Bischoff, A., 2010. Formation and evolution of the highly unconsolidated asteroid 2008 TC3. Meteor. Planet. Sci. Suppl. 45, A83.

Housen, K.R., Holsapple, K.A., Voss, M.E., 1999. Compaction as the origin of the unusual craters on the asteroid Mathilde. Nature 402, $155-157$.

Ilyushin, Y.A., Hagfors, T., Kunitsyn, V.E., 2003. Cometary surface layer properties: possible approaches to radio sounding retrieval during the CONSERT experiment-Numerical simulation and discussion. Radio Sci. 38 (1).

Jacobson, S.A., Scheeres, D.J., 2011. Dynamics of rotationally fissioned asteroids: source of observed small asteroid systems. Icarus 214, 161178. https://doi.org/10.1016/j.icarus.2011.04.009.

Jenniskens, P., Shaddad, M.H., Numan, D., Elsir, S., Kudoda, A.M., Zolensky, M.E., Le, L., Robinson, G.A., Friedrich, J.M., Rumble, D., Steele, A., Chesley, S.R., Fitzsimmons, A., Duddy, S., Hsieh, H.H., Ramsay, G., Brown, P.G., Edwards, W.N., Tagliaferri, E., Boslough, M.B., Spalding, R.E., Dantowitz, R., Kozubal, M., Pravec, P., Borovicka, J., Charvat, Z., Vaubaillon, J., Kuiper, J., Albers, J., Bishop, J.L., Mancinelli, R.L., Sandford, S.A., Milam, S.N., Nuevo, M., Worden, S.P., 2009. The impact and recovery of asteroid 2008 TC3. Nature 458, 485-488. https://doi.org/10.1038/nature07920.

Jurgens, R.F., Goldstein, R.M., Rumsey, H.R., Green, R.R., 1980. Images of Venus by three-station radar interferometry-1977 results. J. Geophys. Res. 85, 8282-8294. https://doi.org/10.1029/ JA085iA13p08282.

Jutzi, M., Holsapple, K., Wünneman, K., Michel, P., 2015. Modeling asteroid collisions and impact processes. In: Michel, P., DeMeo, F.E., Bottke, W.F. (Eds.), Asteroids IV. Univ. Arizona Press, Tucson, pp 341-354. doi:https://doi.org/10.2458/azu_uapress_9780816532131ch018.

Kofman, W., Barbin, Y., Klinger, J., Levasseur-Regourd, A.-C., Barriot, J.-P., Herique, A., Hagfors, T., Nielsen, E., Grün, E., Edenhofer, P., Kochan, H., Picardi, G., Seu, R., van Zyll, J., Elachi, Ch., Melosh, J., Veverka, J., Weissman, P., Svedhem, L.H., Hamran, S.E., Williams, I. P., 1998. Comet nucleus sounding experiment by radiowave transmission. Adv. Space Res. 21, 1589-1598.

Kofman, W., Herique, A., Barbin, Y., Barriot, J.-P., Ciarletti, V., Clifford, S., Edenhofer, P., Elachi, C., Eyraud, C., Goutail, J.-P., Heggy, E., Jorda, L., Lasue, J., Levasseur-Regourd, A.-C., Nielsen, E., Pasquero, P., Preusker, F., Puget, P., Plettemeier, D., Rogez, Y., Sierks, H., 
Statz, Svedhem, H., Williams, I., Zine, S., Van Zyl, J., 2015. Properties of the 67P/Churyumov-Gerasimenko interior revealed by CONSERT radar. Science 349 (aab0639), 1-6. https://doi.org/10.1126/science. aab0639.

Kofman, W., Herique, A., Goutail, J.P., Consert team, 2004. Consert Experiment: Description and Performances in View of the New Target. Springer, Dordrecht.

Kofman, W., Herique, A., Goutail, J.-P., Hagfors, T., Williams, I.P., Nielsen, E., Barriot, J.-P., Barbin, Y., Elachi, C., Edenhofer, P., Levasseur-Regourd, A.-C., Plettemeier, D., Picardi, G., Seu, R. Svedhem, V., 2007. The Comet Nucleus Sounding Experiment by Radiowave Transmission (CONSERT): a short description of the instrument and of the commissioning stages. Space Sci. Rev. 128, $413-$ 432. https://doi.org/10.1007/s11214-006-9034-9.

Kraus, J.D., 1986. Radio Astronomy, 1986. Cygnus-Quasar Books, Powell, Ohio.

Lauretta, D.S., 2015. OSIRIS-REx asteroid sample-return mission. In: Pelton, J.N., Allahdadi, F. (Eds.), Handbook of Cosmic Hazards and Planetary Defense. Springer International Publishing, Cham, pp. 543567. doi:https://doi.org/10.1007/978-3-319-03952-7_44.

Lowry, S.C., Weissman, P.R., Duddy, S.R., Rozitis, B., Fitzsimmons, A., Green, S.F., Hicks, M.D., Snodgrass, C., Wolters, S.D., Chesley, S.R., Pittichová, J., van Oers, P., 2014. The internal structure of asteroid (25143) Itokawa as revealed by detection of YORP spin-up. Astron. Astrophys. 562 (A48), 1-9. https://doi.org/10.1051/0004-6361/ 201322602.

Magri, C., Nolan, M.C., Ostro, S.J., Giorgini, J.D., 2007. A radar survey of main-belt asteroids: Arecibo observations of 55 objects during 1999-2003. Icarus 186, 126-151. https://doi.org/10.1016/j. icarus.2006.08.018.

Margot, J.L., Nolan, M.C., Benner, L.a.M., et al., 2002. Binary asteroids in the near-earth object population. Science 296, 1445-1448. https:// doi.org/10.1126/science.1072094.

Michel, P., Benz, W., Tanga, P., Richardson, D.C., 2001. Collisions and gravitational reaccumulation: forming asteroid families and satellites. Science 294, 1696-1700. https://doi.org/10.1126/science.1065189.

Michel, P., Richardson, D.C., 2013. Collision and gravitational reaccumulation: possible formation mechanism of the asteroid Itokawa. Astron. Astrophys. 554 (L1), 1-29.

Michel, P., Cheng, A., Küppers, M., Pravec, P., Blum, J., Delbo, M., Green, S.F., Rosenblatt, P., Tsiganis, K., Vincent, J.B., Biele, J., Ciarletti, V., Hérique, A., Ulamec, S., Carnelli, I., Galvez, A., Benner, L., Naidu, S.P., Barnouin, O.S., Richardson, D.C., Rivkin, A., Scheirich, P., Moskovitz, N., Thirouin, A., Schwartz, S.R., Campo Bagatin, A., Yu, Y., 2016. Science case for the Asteroid Impact Mission (AIM): a component of the Asteroid Impact \& Deflection Assessment (AIDA) mission. Adv. Space Res. 57, 2529-2547. https:// doi.org/10.1016/j.asr.2016.03.031.

Michel, P., Kueppers, M., Sierks, H., Carnelli, I., Cheng, A.F., Mellab, K., Granvik, M., Kestila, A., Kohout, T., Muinonen, K., Nasila, A., Penttila, A., Tikka, T., Tortora, P., Ciarletti, V., Herique, A., Murdoch, N., Asphaug, E., Rivkin, A., Barnouin, O., Campo Bagatin, A., Pravec, P., Richardson, D.C., Schwartz, S.R., Tsiganis, K., Ulamec, S., Karatekin, O., 2018. European component of the AIDA mission to a binary asteroid: Characterization and interpretation of the impact of the DART mission. Adv. Space Res. 62, 2261-2272.

Morbidelli, A., Chambers, J., Lunine, J.I., Petit, J.M., Robert, F., Valsecchi, G.B., Cyr, K.E., 2000. Source regions and timescales for the delivery of water to the Earth. Meteorit. Planet. Sci. 35, 1309-1320.

Mouginot, J., Pommerol, A., Kofman, W., Beck, P., Schmitt, B., Herique, A., Grima, C., Safaeinili, A., Plaut, J.J., 2010. The 3-5MHz global reflectivity map of Mars by MARSIS/Mars Express: implications for the current inventory of subsurface H2O. Icarus 210, 612-625. https:// doi.org/10.1016/j.icarus.2010.07.003.

Murdoch, N., Sánchez, P., Schwartz, S.R., Miyamoto, H., 2015. Asteroid surface geophysics. In: Michel, P., DeMeo, F.E., Bottke, W.F. (Eds.), Asteroids IV. University of Arizona Press, Tucson, pp 767-792. doi: https://doi.org/10.2458/azu_uapress_9780816532131-ch039.
Naidu, S.P., Benner, L.A.M., Margot, J.-L., Busch, M.W., Taylor, P.A., 2016. Capabilities of Earth-based radar facilities for near-earth asteroid observations. Astron. J. 152 (99). https://doi.org/10.3847/ 0004-6256/152/4/99, 1-29.

Nakamura, T., Noguchi, T., Tanaka, M., Zolensky, M.E., Kimura, M., Tsuchiyama, A., Nakato, A., Ogami, T., Ishida, H., Uesugi, M., Yada, T., Shirai, K., Fujimura, A., Okazaki, R., Sandford, S.A., Ishibashi, Y., Abe, M., Okada, T., Ueno, M., Mukai, T., Yoshikawa, M., Kawaguchi, J., 2011. Itokawa dust particles: a direct link between stype asteroids and ordinary chondrites. Science 333, 1113-1116. https://doi.org/10.1126/science.1207758.

Nozette, S., Spudis, P., Bussey, B., Jensen, R., Raney, K., Winters, H., Lichtenberg, C.L., Marinelli, W., Crusan, J., Gates, M., Robinson, M., 2010. The lunar reconnaissance orbiter miniature radio frequency (Mini-RF) technology demonstration. Space Sci. Rev. 150, 285-302. https://doi.org/10.1007/s11214-009-9607-5.

Oberst, Jürgen, Wickhusen, K., Willner, K., Gwinner, K., Spiridonova, S., Kahle, R., et al., 2018. DePhine - The Deimos and Phobos Interior Explorer. Adv. Space Res. 62, 2220-2238.

Olhoeft, G.R., 1979. Tables of Room Temperature Electrical Properties for Selected Rocks and Minerals with Dielectric Permittivity Statistics. USGS Report.

Ostro, S.J., Margot, J.-L., Benner, L.A.M., Giorgini, J.D., Scheeres, D.J., Fahnestock, E.G., Broschart, S.B., Bellerose, J., Nolan, M.C., Magri, C., Pravec, P., Scheirich, P., Rose, R., Jurgens, R.F., De Jong, E.M., Suzuki, S., 2006. Radar imaging of binary near-earth asteroid (66391) 1999 KW4. Science 314, 1276-1280. https://doi.org/ 10.1126/science. 1133622.

Ostro, S.J., Scott, R., Hudson, Nolan., et al., 2000. Radar observations of asteroid 216 Kleopatra. Science 288, 836-839. https://doi.org/ 10.1126/science.288.5467.836.

Pasquero, O.P., Hérique, A., Kofman, W., 2017. Oversampled pulse compression based on signal modeling: application to CONSERT/ rosetta radar. IEEE Trans. Geosci. Remote Sens. 55, 2225-2238. https://doi.org/10.1109/TGRS.2016.2639449.

Prettyman, T.H., Yamashita, N., Toplis, M.J., McSween, H.Y., Schörghofer, N., Marchi, S., Feldman, W.C., Castillo-Rogez, J., Forni, O., Lawrence, D.J., Ammannito, E., Ehlmann, B.L., Sizemore, H.G., Joy, S.P., Polanskey, C.A., Rayman, M.D., Raymond, C.A., Russell, C.T., 2017. Extensive water ice within Ceres' aqueously altered regolith: evidence from nuclear spectroscopy. Science 355, 55-59. https://doi. org/10.1126/science.aah6765.

Pursiainen, S., Kaasalainen, M., 2014. Detection of anomalies in radio tomography of asteroids: source count and forward errors. Planet. Space Sci. 99, 36-47. https://doi.org/10.1016/j.pss.2014.04.017.

Richardson, J.E., 2011. Regolith generation, retention, and movement on asteroid surfaces: early modeling results, Presentation at the 42nd Lunar and Planetary Science Conference, 7-11 March 2011, The Woodlands, Texas. LPI Contribution No. 1608, p.1084.

Rubincam, D.P., 2000. Radiative spin-up and spin-down of small asteroids. Icarus 148, 2-11. https://doi.org/10.1006/icar.2000.6485.

Russell, C.T., McSween, H.Y., Jaumann, R., Raymond, C.A., 2015. The dawn mission to vesta and ceres. A. In: Michel, P., DeMeo, F.E., Bottke, W.F. (Eds.), Asteroids IV. Univ. Arizona Press, Tucson, pp. 419-432. doi:https://doi.org/10.2458/azu_uapress_9780816532131-ch022.

Scheeres, D.J., Gaskell, R.W., 2008. Effect of density inhomogeneity on YORP: The case of Itokawa. Icarus 198, 125-129. https://doi.org/ 10.1016/j.icarus.2008.07.010.

Schorghofer, N., 2008. The lifetime of ice on main belt asteroids. Astrophys. J. 682, 697-705. https://doi.org/10.1086/588633.

Shepard, M.K., Taylor, P.A., Nolan, M.C., Howell, E.S., Springmann, A., Giorgini, J.D., Warner, B.D., Harris, A.W., Stephens, R., Merline, W. J., Rivkin, A., Benner, L.A.M., Coley, D., Clark, B.E., Ockert-Bell, M., Magri, C., 2015. A radar survey of M- and X-class asteroids. III. Insights into their composition, hydration state, \& structure. Icarus 245, 38-55. https://doi.org/10.1016/j.icarus.2014.09.016.

Sihvola, A.H., 1999. Electromagnetic Mixing Formulas and Applications. IET. 
Sihvola, A.H., Lindell, I.V., 1992. Effective permeability of mixtures. In: Priou, A. (Ed.), Progress in Electromagnetics Research (PIER 6), Dielectric Properties of Heterogeneous Materials, Elsevier, Amsterdam.

Simpson, R, 2007. In: Willis, N.J., Griffiths, H.D. (Eds.), Planetary Exploration in Advances in bistatic radar. SciTech Publishing Inc., Raleigh NC.

Slade, M.A., Benner, L.A.M., Silva, A., 2011. Goldstone solar system radar observatory: earth-based planetary mission support and unique science results. Proc. IEEE 99, 757-769. https://doi.org/10.1109/ JPROC.2010.2081650.

Snodgrass, C., Jones, G.H., Boehnhardt, H., Gibbings, A., Homeister, M., Andre, N., Beck, P., Bentley, M.S., Bertini, I., Bowles, N., Capria, M. T., Carr, C., Ceriotti, M., Coates, A.J., Della Corte, V., Donaldson Hanna, K.L., Fitzsimmons, A., Gutierrez, P.J., Hainaut, O.R., Herique, A., Hilchenbach, M., Hsieh, H.H., Jehin, E., Karatekin, O., Kofman, W., Lara, L.M., Laudan, K., Licandro, J., Lowry, S.C., Marzari, F., Masters, A., Meech, K.J., Moreno, F., Morse, A., Orosei, R., Pack, A., Plettemeier, D., Prialnik, D., Rotundi, A., Rubin, M., Sanchez, J.P., Sheridan, S., Trieloff, M., Winterboer, A., 2018, The Castalia mission to Main Belt Comet 133P/Elst-Pizarro. Adv. Space Res. 62, 1947-1976.

Stillman, D., Olhoeft, G., 2008. Frequency and temperature dependence in electromagnetic properties of Martian analog minerals. J. Geophys. Res. 113, 1-14. https://doi.org/10.1029/2007JE002977.

Tholen, D.J., 1989. Asteroid taxonomic classifications. In: Binzel, R.P., Gehrels, T., Mathews, M.S. (Eds.), Asteroids II. University of Arizona Press, Tucson, pp. 1139-1150.

Thompson, T.W., Ustinov, E.A., Heggy, E., 2011. Modeling radar scattering from icy lunar regoliths at $13 \mathrm{~cm}$ and $4 \mathrm{~cm}$ wavelengths. J. Geophys. Res. 116, 1-27. https://doi.org/10.1029/2009JE003368.

Tsuda, Y., Nakazawa, S., Kushiki, K., Yoshikawa, M., Kuninaka, H., Watanabe, S., 2016. Flight status of robotic asteroid sample return mission Hayabusa2. Acta Astronaut. 127, 702-709. https://doi.org/ 10.1016/j.actaastro.2016.01.027.

Ulamec, S., Biele, J., 2015. Relevance of PHILAE and MASCOT in-situ Investigations for Planetary Defense. Presentation at the 4th IAA Planetary Defense Conference, Frascati, 2015.

Ulander, L.M., Frolind, P.-O., 1998. Ultra-wideband SAR interferometry. IEEE Trans. Geosci. Remote Sens. 36, 1540-1550.
Vernazza, P., Beck, P., 2016. Composition of solar system small bodies. In: Elkins-Tanton, Linda T., Weiss, Benjamin P., (Eds.). Planetesimals: Early Differentiation and Consequences for Planets, Cambridge Univ. Press. Availablr from: arXiv:1611.08731.

Wahlund, Jan-Erik, Andrews, D., De Sanctis, M.C., Futaana, Y., Herique, A., Ivchenko, N., Retherford, K., Simon, S., Thomas, N., Tortora, P., J. Trigo-Rodriguez et Heavy Metal team, 2016, Heavy Metal, Response to a Call for a Medium-Size Mission Opportunity in ESA's Science Programme for launch in 2029 (M5).

Walsh, K.J., Richardson, D.C., Michel, P., 2008. Rotational breakup as the origin of small binary asteroids. Nature 454, 188-191. https://doi. org/10.1038/nature07078.

Yeomans, D.K., Barriot, J.-P., Dunham, D.W., Farquhar, R.W., Giorgini, J.D., Helfrich, C.E., Konopliv, A.S., McAdams, J.V., Miller, J.K., Owen, W.M., Scheeres, D.J., Synnott, S.P., Williams, B.G., 1997. Estimating the mass of asteroid 253 Mathilde from tracking data during the NEAR flyby. Science 278, 2106-2109.

Zhu, M.-H., Fa, W., Ip, W.-H., Huang, J., Liu, T., Meng, L., Yan, J., Xu, A., Tang, Z., Wang, X., Qiao, D., 2014. Morphology of asteroid (4179) Toutatis as imaged by Chang'E-2 spacecraft: Zhu et al.: Morphology of Asteroid (4179) Toutatis. Geophys. Res. Lett. 41, 328-333. https:// doi.org/10.1002/2013GL058914.

Zolensky, M.E., Zega, T.J., Yano, H., Wirick, S., Westphal, A.J., Weisberg, M.K., Weber, I., Warren, J.L., Velbel, M.A., Tsuchiyama, A., Tsou, P., Toppani, A., Tomioka, N., Tomeoka, K., Teslich, N., Taheri, M., Susini, J., Stroud, R., Stephan, T., Stadermann, F.J., Snead, C.J., Simon, S.B., Simionovici, A., See, T.H., Robert, F., Rietmeijer, F.J.M., Rao, W., Perronnet, M.C., Papanastassiou, D.A., Okudaira, K., Ohsumi, K., Ohnishi, I., Nakamura-Messenger, K., Nakamura, T., Mostefaoui, S., Mikouchi, T., Meibom, A., Matrajt, G., Marcus, M.A., Leroux, H., Lemelle, L., Le, L., Lanzirotti, A., Langenhorst, F., Krot, A.N., Keller, L.P., Kearsley, A.T., Joswiak, D., Jacob, D., Ishii, H., Harvey, R., Hagiya, K., Grossman, L., Grossman, J.N., Graham, G.A., Gounelle, M., Gillet, P., Genge, M.J., Flynn, G., Ferroir, T., Fallon, S., Ebel, D.S., Dai, Z.R., Cordier, P., Clark, B., Chi, M., Butterworth, A.L., Brownlee, D.E., Bridges, J.C., Brennan, S., Brearley, A., Bradley, J.P., Bleuet, P., Bland, P.A., Bastien, R., 2006. Mineralogy and petrology of Comet 81P/Wild 2 nucleus samples. Science 314, 1735-1739. https://doi.org/ 10.1126/science. 1135842 . 